\title{
Energy master equation: A low-temperature approximation to Bässler's random-walk model
}

\author{
Jeppe C. Dyre \\ Institute of Mathematics and Physics (IMFUFA), Roskilde University, P.O. Box 260, DK-4000 Roskilde, Denmark
}

(Received 20 October 1994)

\begin{abstract}
The first part of this paper deals with the justification of Bässler's phenomenological random-walk model for viscous liquids [Phys. Rev. Lett. 58, 767 (1987)], which considers the random walk of a "particle" representing the liquid state on a $d$-dimensional infinite cubic lattice with site energies chosen randomly according to a Gaussian. The random-walk model is here derived from Newton's laws by making a number of simplifying assumptions. In the second part of the paper an approximate low-temperature description of energy fluctuations in the random-walk model-the energy master equation (EME)-is arrived at. The EME is one dimensional and involves only energy; it is derived by arguing that percolation dominates the relaxational properties of the random-walk model at low temperatures. The approximate EME description of the random-walk model is expected to be valid at low temperatures at long times in high dimensions. However, computer simulations show that the EME works well already in two dimensions and at only moderately low temperatures. The EME has no randomness and no fitting parameters. The EME is completely specified from the density of states and the attempt frequency of the randomwalk model. The EME allows a calculation of the energy probability distribution at realistic laboratory time scales for an arbitrarily varying temperature as function of time. The EME is probably the only realistic equation available today with this property that is also explicitly consistent with statistical mechanics. The final part of the paper gives a comprehensive discussion, comparing the EME to related work and listing the EME's qualitatively correct predictions, its new predictions, and some "wrong" predictions, most of which go against the common picture of viscous liquids and the glass transition without violating experiments.
\end{abstract}

\section{INTRODUCTION}

The glass transition takes place when a liquid upon cooling becomes more and more viscous and finally solidifies to form a glassy solid. ${ }^{1-14}$ Most, or perhaps all, liquids are able to form glasses when cooled sufficiently fast to avoid crystallization. Examples of glasses include the classical oxide glasses, ${ }^{15}$ ionic glasses, ${ }^{16}$ polymers, ${ }^{6,17,18}$ metallic glasses, ${ }^{19}$ and glasses made by cooling organic liquids to low temperatures. ${ }^{20,21}$ Even simple liquids form glasses in computer experiments, where extremely high cooling rates are possible. ${ }^{12,22,23}$ Spin glasses are examples of nonliquid systems that exhibit glassy features. ${ }^{24,25}$

The glass transition is still far from well understood, but the kinetic nature of the transition is not in doubt. The glass transition is not a phase transition, though it is thermodynamically similar to a second-order phase transition. This is evidenced by several facts universally observed: The transition is not sharp, the transition temperature depends on the cooling rate, and the transition is irreversible and exhibits various hysteresis phenomena.

Despite large chemical differences, viscous liquids close to the glass transition have common features, notably a broad distribution of relaxation times and a stronger than Arrhenius temperature dependence of the viscosity. Around the glass transition there are further common characteristics like the overshoot of the specific heat upon reheating, ${ }^{8,14}$ the crossover effect, ${ }^{8}$ or the prepeak upon the melting of a well-annealed glass. ${ }^{11}$ The universal properties of viscous liquids and the glass transition motivates a search for a phenomenological model valid for any viscous liquid.

While phenomenological models of viscous liquids and the glass transition have been studied for many years, the 1980's brought an interesting first-principles theory, the mode-coupling theory. ${ }^{26,27}$ Extensive work has gone into studying the mode-coupling theory and comparing it to experiment. At present there seems to be a growing consensus $^{28}$ that mode-coupling theory gives an accurate description of the onset of viscous behavior, the temperature region where the relaxation times are shorter than about $1 \mathrm{~ns}$. However, the theory does not seem to be able to explain the highly viscous regime and the laboratory glass transition. This is because the activated "hopping" type processes that dominate this regime are not accounted for in the simplest version of mode-coupling theory, ${ }^{26-30}$ but have to be postulated as an extra assumption. Thus, the focus is now once again on attempts to formulate a phenomenological model that captures the essentials of viscous liquids and the glass transition. Nevertheless, mode-coupling theory has served to emphasize the different physical bases of the low- and hightemperature regimes.

Since the glass transition is a kinetic "freezing" of the viscous liquid, a phenomenological model should first of all incorporate the basic physics of viscous liquids in thermal equilibrium. An important characteristic here is the average relaxation time of the viscous liquid $\tau$, which is a direct measure of the time needed for molecular rearrangements. The average relaxation time may be determined, e.g., as the inverse dielectric, mechanical or 
specific-heat loss-peak frequency. Alternatively, it may be calculated from the viscosity $\eta$ and the infinite frequency shear modulus $G_{\infty}$ by means of the expression

$$
\tau=\frac{\eta}{G_{\infty}}
$$

These definitions do not give exactly identical $\tau$ 's, ${ }^{13}$ but the difference is insignificant for the present discussion. Typical values of $\tau$ for glass-forming liquids lie in the millisecond, second or even hour range. These times are to be compared to a typical microscopic time, the average vibration time, which is less than 1 psec.

The basic thing one would like to understand about the average relaxation time is its non-Arrhenius temperature dependence. For almost all viscous liquids $\tau$ has an apparent activation energy that increases as the temperature decreases. Naive models assuming some distribution of energy barriers usually lead to the opposite behavior. Therefore, explaining $\tau(T)$ is a real challenge, but also a likely key to understanding viscous liquids.

The phenomenological models may be classified into two types (an alternative to the below classification has been given by Scherer in an excellent review of relaxation in viscous liquids ${ }^{29}$ ). One type of models, "type-I," are models that have a non-Arrhenius average relaxation time, but otherwise do not attempt to model the liquid. These models are so simple that they can be analyzed in detail. ${ }^{31}$ Examples of type-I models are Derrida's random energy model, ${ }^{32}$ the kinetic Ising model, ${ }^{33}$ or the tiling model of Weber, Fredrickson, and Stillinger. ${ }^{34}$ The other type of models, "type-II," do attempt to realistically model the physics of real viscous liquids. In all type-II models the elementary flow process occurs within a "cooperatively rearranging region." The type-II models can be further classified according to which thermodynamical quantity controls $\tau$, entropy, volume, or energy.

A well-known entropy-controlled model is the theory of Gibbs and co-workers. ${ }^{35,36}$ According to this model the average relaxation time is expressed in terms of the excess configurational entropy $S_{c}$ as $\tau \propto \exp \left[C /\left(T S_{c}\right)\right]$. The model correlates the non-Arrhenius behavior with the Kauzmann paradox, ${ }^{2,11}$ the fact that the configurational entropy extrapolates to zero at a finite temperature, $T_{K}$ : Expanding $S_{c}$ to first order close to $T_{K}$, the average relaxation time follows the VogelFulcher-Tammann (VFT) law ${ }^{11,29}$ (where $A$ is a constant and the characteristic temperature $T_{0}$ is predicted to be equal to $T_{K}$ ):

$$
\tau=\tau_{0} e^{A /\left(T-T_{0}\right)}
$$

The VFT law gives a good fit to $\tau(T)$ for many viscous liquids and in most experiments one also finds that $T_{0}$ is indeed close to $T_{K}{ }^{11}$

Gibbs' model predicts that underlying the laboratory glass transition there is a genuine second-order phase transition at $T=T_{K}$ to a state of zero configurational entropy. However, there are a number of problems with this approach. The original Gibbs-DiMarzio model ${ }^{35}$ was based on a mean-field theory for polymers that later was shown to be incorrect. ${ }^{37}$ Also, the VFT law seldom applies in the whole temperature range of interest; usually deviations occur close to the glass transition where the average relaxation time is less temperature dependent than predicted. ${ }^{8,20,29}$ [If the physics of the high and low viscosity regimes are different, as predicted by modecoupling theory, there is no motivation to choose a phenomenological representation of $\tau(T)$ which can cross the boundary between the two regimes.] Finally, it should be noted that the Kauzmann paradox does not have to be a paradox. As shown by Angell and Rao in $1972,{ }^{38}$ even a system with only two energy levels has an entropy which, if only known at high temperatures, extrapolates to zero at a positive temperature. Though this model does not fit experiment, the excess entropy data may be fitted with a model with only a finite number of energy levels and thus a positive entropy at any positive temperature. ${ }^{39}$

The standard example of a volume-controlled model is the "free volume model." 40 In this model, the average relaxation time is determined by the volume freely available for cooperative rearrangements of the molecules, $V_{f}$, according to the expression $\tau \propto \exp \left(C / V_{f}\right)$. In the simplest version of the model the free volume decreases linearly with decreasing temperature, leading if $V_{f}=0$ at $T=T_{0}$ to a non-Arrhenius $\tau(T)$ of the VFT type [Eq. (2)].

In energy-controlled models one formulates a master equation $^{41}$ governing the dynamics of the cooperatively rearranging regions. The relevance of potential energy was previously emphasized in 1969 in a classic paper by Goldstein. ${ }^{42}$ More recently, Brawer proposed a model where transitions between different states occur via excitations to a common high-lying energy level. ${ }^{8,43}$ This picture goes back to Goldstein. ${ }^{39}$ Brawer's model was later simplified. ${ }^{44}$

Bässler's random-walk model ${ }^{45,46}$ is an energycontrolled model, which is similar to those used in the description of ac conduction in disordered solids ${ }^{47,48}$ and of energetic relaxation and diffusion of electronic excitations in random organic solids. ${ }^{49}$ The model considers the random walk of a "particle" on a cubic lattice in $d$ dimensions, where each site has an energy chosen randomly according to a Gaussian. The particle represents the state of a cooperatively rearranging region. For the random-walk model $\tau(T)$ is predicted ${ }^{45,46}$ to follow

$$
\tau=\tau_{0} \exp \left[\frac{C}{T^{2}}\right] .
$$

This simple expression fits experiments well. ${ }^{45,46,50,51}$ An even better fit is obtained by using the following generalization of Eq. (3): $\tau=\tau_{0} \exp \left(C / T^{n}\right) .{ }^{20,21,52}$

In a recent paper by Arkhipov and Bässler ${ }^{53}$ the random-walk model was extended to a model that reduces to the original model at high temperatures - the "real liquid" regime-while at low temperatures-the "supercooled melt" regime - the system is described by a simple master equation. The $\mathrm{idea}^{53}$ is that, at high temperatures direct jumps between metastable states are possible because the energy landscape itself fluctuates; these jumps correspond to an elementary step on the $d$ - 
dimensional lattice of the original random-walk model. At low temperatures, on the other hand, the landscape fluctuations are frozen on the relevant time scale and each jump leads to a totally new configuration, the dynamics here being described by a simple master equation. ${ }^{44}$

The purpose of the present paper is to show that the master equation, assumed by Arkhipov and Bässler to describe the different physics going on at low temperatures, in fact gives a good description of the low-temperature behavior of the original random-walk model. Thus, in a sense the Arkhipov-Bässler model is contained in Bässler's original and simpler random-walk model. The low-temperature approximate master equation, the "energy master equation" is arrived at by arguing that percolation in the random-walk model becomes important at low temperatures. The transition state energy of the energy master equation is identified with the highest energy met on a percolation path. In effect, one arrives at a picture which is similar to that recently proposed by Hunt, ${ }^{13,54}$ according to which the low-temperature properties of viscous liquids are dominated by percolation. However, in his works effects of cooperativity are treated separately in relaxation and in thermodynamics.

The paper has the following outline. In Sec. II a justification of the random-walk model is given where the model is traced back to Newton's laws for the molecules of a cooperatively rearranging region. This section supplements the original arguments for the model given by Bässler and co-workers. ${ }^{45,46}$ In Sec. III the approximate energy master equation is derived. In Sec. IV computer simulations are presented, comparing the random walk model and the energy master equation. Section $V$ discusses what to expect at the glass transition according to the energy master equation. Section VI gives a comprehensive discussion which includes a qualitative comparison to experiment. Finally, Sec. VII gives the conclusions.

\section{THE RANDOM-WALK MODEL AND ITS “DERIVATION" FROM NEWTON'S SECOND LAW}

The purpose of this section is to "derive" the randomwalk model ${ }^{45,46}$ from the equations of motion for the molecules of the viscous liquid. The "derivation," which proceeds in five steps, is not rigorous, but rather an attempt to make explicit the assumptions that need to be made in order to justify the model from basic principles. The viewpoints presented below are similar to those of Bässler, but there are also some differences as will be discussed at the end of this section.

Before presenting the "derivation" of the random-walk model, we recall the exact definition of the model. The model considers the random walk of a particle on an infinite $d$-dimensional cubic lattice. The particle represents the state of the region, which is thus completely specified by $d$ integer coordinates. Each state has an energy $E$, which is chosen randomly according to a Gaussian with variance $\sigma$ :

$$
n(E)=\frac{1}{\sqrt{2 \pi \sigma^{2}}} \exp \left[-\frac{E^{2}}{2 \sigma^{2}}\right) .
$$

The energies of adjacent states are uncorrelated. The dynamics of the system is described by a master equation, ${ }^{41}$ specifying the time development of the probability that the particle is in state $i, P_{i}$. If $\Gamma(i \rightarrow j)$ is the transition rate for jumps from state $i$ to state $j$, the general master equation $^{41}$ is

$$
\frac{d P_{i}}{d t}=-\sum_{j} \Gamma(i \rightarrow j) P_{i}+\sum_{j} \Gamma(j \rightarrow i) P_{j} .
$$

The first term describes particles jumping away from state $i$ and the second term describes particles jumping into state $i$. The jump rates must satisfy the principle of detailed balance, ${ }^{41}$ which ensures consistency with statistical mechanics $\left[\beta=1 /\left(k_{B} T\right)\right]$,

$$
\frac{\Gamma(i \rightarrow j)}{\Gamma(j \rightarrow i)}=\exp \left[\beta\left(E_{i}-E_{j}\right)\right]
$$

In random-walk models the transition rates are usually chosen to be zero except for nearest-neighbor jumps (i.e., where a single coordinate changes plus or minus one). If $\Gamma_{0}$ is the "attempt frequency," the transition rate for a nearest-neighbor jump is in Bässler's random-walk model given by Metropolis dynamics,

$$
\Gamma(i \rightarrow j)=\left\{\begin{array}{l}
\Gamma_{0}, \quad E_{i}>E_{j} \\
\Gamma_{0} e^{-\beta\left(E_{j}-E_{i}\right)}, \quad E_{i}<E_{j} .
\end{array}\right.
$$

It is realistic to take $\Gamma_{0}$ to be of order $10^{13} \mathrm{~Hz}$, corresponding to a typical phonon frequency. Note that Eq. (7) satisfies the principle of detailed balance Eq. (6).

What kind of predictions can be made from the random-walk model? The model predicts how the average energy changes in time for any externally controlled time-dependent temperature. This includes monitoring how the energy relaxes to equilibrium from a nonequilibrium state, or how the dynamic specific heat changes through the glass transition. In particular, the average relaxation time for energy relaxations close to equilibrium can be calculated as a function of temperature, and the equilibrium frequency-dependent specific heat may be obtained.

We now proceed to justify the random-walk model from basic principles in five steps, assuming that the molecules of the viscous liquid are described by classical mechanics.

Step 1: The Region Assumption. All type-II models for the dynamics of viscous liquids assume cooperative "flow events" that are localized to small "regions" of the liquid. $6,8,36,39,42-44,55-60$ These regions have been called "cooperatively rearranging subsystems", 29 or "cooperatively rearranging regions,"36 "quasi-independent units,"39 "thermokinetic structures,"58 "molecular domains," 59 or "dynamically correlated domains.",60 This picture of viscous flow, proceeding via strongly cooperative motion of particles confined to small regions of the liquid, has been confirmed by computer simulations. ${ }^{61,62}$ The "region assumption," however, is not just the quite reasonable idea that flow events are localized. The assumption is the much stronger one that the liquid 
may be regarded as an ensemble of noninteracting regions. There are two potential problems with this assumption. It ignores region-region interactions that may be important because the regions are expected to be relatively small [some 10-20 $\AA$ (Ref. 58)]. Also, the picture is static and not easy to relate to an actual flow that will deform the regions. Nevertheless, the region assumption seems to be necessary to arrive at a simple phenomenological model.

Step 2: Replacing Newton's laws with Langevin dynamics. From now on the attention is confined to a single region, the molecules of which move according to Newton's laws. The motion depends on the potential energy as function of the molecular coordinates, $U\left(Q_{1}, \ldots, Q_{d}\right)$ (for simplicity only rectangular coordinates are considered). The importance of the potential energy "surface" for understanding viscous liquids and the glass transition has been emphasized in a number of papers. ${ }^{11,30,39,42,63,64}$ Following the tradition in polymer physics, ${ }^{65}$ we now replace Newton's deterministic equations of motion by stochastic Langevin equations (similar nondeterministic equations are used for the description of Brownian particles suspended in liquids ${ }^{66}$ ). The Langevin equations of motion ${ }^{41,65}$ are

$$
\dot{Q}_{i}=-\mu \frac{\partial U}{\partial Q_{i}}+\xi_{i}(t) \quad(i=1, \ldots, d),
$$

where $\mu$ is the "mobility" [velocity/force] and $\xi_{i}(t)$ is a Gaussian white-noise term obeying

$$
\left\langle\xi_{i}(t) \xi_{j}\left(t^{\prime}\right)\right\rangle=2 \mu k_{B} T \delta_{i, j} \delta\left(t-t^{\prime}\right) .
$$

The crucial property of the Langevin equations of motion $^{41,65}$ is that each state is visited with the correct canonical probability $P_{0}$ of statistical mechanics,

$$
P_{0}\left(Q_{1}, \ldots, Q_{d}\right) \propto e^{-\beta U\left(Q_{1}, \ldots, Q_{d}\right)} .
$$

Physically, the assumption of Langevin dynamics is reasonable for viscous liquids, ${ }^{67}$ because the molecules collectively vibrate in potential energy minima for long times before occasionally "jumping" to another potential energy minimum. And the rate of jumps between two energy minima is, for both Newtonian and Langevin dynamics, dominated by a factor $\propto \exp (-\beta \Delta U),{ }^{68}$ where $\Delta U$ is the energy barrier to be overcome.

Step 3: From Langevin dynamics to a hopping model. We now proceed to discretize the spatial variables of the Langevin equations. The resulting state space is a $d$ dimensional cubic lattice. It is reasonable to assume that, since the underlying Langevin dynamics has a continuous trajectory, only nearest-neighbor jumps are allowed on the lattice. For the jump rates those given by Eq. (7) are an obvious choice: Unless infinitely steep potentials are allowed, the Langevin equation implies that it takes some definite (but very small) time to travel the discretization length downhill; in the discrete version this means there should be a maximum jump rate. If the discretization is to be self-consistent, the jump rates must be uniquely determined from the state energies. The simplest jump rates satisfying these conditions are the Metropolis rates [Eq. (7)]. These jump rates ignore the possible existence of a barrier to be overcome between two nearest-neighbor discrete states. The discretization does not allow continuous vibrational motion. The term "energy" $E$ is henceforth to be thought of as the potential energy $U$ at a minimum or a saddle point, the "configurational part" of the potential energy. Similarly, the term "specific heat" refers to the configurational part of the measured specific heat, the so-called "excess specific heat" (in excess of the phonon contribution to the specific heat).

Step 4: Replacing complexity with randomness. The potential energy is a very complex function with numerous minima. ${ }^{30,42,63}$ Therefore, it is reasonable to replace the function $E$ defined on the lattice with a function that is in some sense random. The basic idea of replacing complexity with randomness ${ }^{69}$ is that some phenomena occurring in a specific complex system are typical of those that occur in most systems chosen randomly out of an ensemble of possible systems. If this is so, the study of random systems tells us what to expect for particular complex systems. Motion in random potentials has been studied extensively in various contexts. ${ }^{47,48,70,71}$ In discretizing such a model one often chooses a discretization length $a$ equal to the correlation length of the random function and assumes that correlations beyond $a$ may be ignored. ${ }^{71}$ When this is done for the hopping model arrived at above, the values of the potential are assumed to be uncorrelated from point to point on the $d$-dimensional cubic lattice. In this approximation the model is completely specified by the energy probability distribution, the "density of states" $n(E)$.

Step 5: The assumption of cooperativity. A region contains many molecules and thus $d \gg 1$. Any system with many degrees of freedom has a density of states for which the entropy as function of energy, $S(E)=\ln [n(E)]$, at relevant energies ${ }^{72}$ obeys

$$
\frac{\partial S}{\partial E}>0 ; \quad \frac{\partial^{2} S}{\partial E^{2}}<0 \text {. }
$$

The Gaussian Eq. (4) obeys Eq. (11), but only for negative energies. However, at any temperature negative energies are most likely for the Gaussian, and therefore this density of states is permissible as representing a system with many degrees of freedom.

The assumption of a Gaussian density of states concludes the "derivation" of Bässler's random-walk model. The model is completely specified by the parameters $\Gamma_{0}$, $\sigma$, and $d$. The first two are scaling parameters, so from a qualitative point of view only the dimension $d$ is of interest.

In thermal equilibrium the probability of visiting any given site is given by the Boltzmann factor $\exp (-\beta E)$. Combining this with the Gaussian probability Eq. (4), one finds for the equilibrium energy probability distribution $P_{0}(E) \propto \exp \left[-\beta E-E^{2} /\left(2 \sigma^{2}\right)\right]$. By "completing the square" and normalizing, one finds

$P_{0}(E)=\frac{1}{\sqrt{2 \pi \sigma^{2}}} \exp \left[-\frac{(E-\bar{E})^{2}}{2 \sigma^{2}}\right], \quad \bar{E}=-\sigma^{2} \beta$.

Clearly $\bar{E}$ is the average energy. Note that $\bar{E}$ is also the most likely energy (in fact, for any system with many de- 
grees of freedom the average energy is close to the most likely energy). The equilibrium specific heat $c_{0}$ is given by

$$
c_{0} \equiv \frac{d \bar{E}}{d T}=\frac{\sigma^{2}}{k_{B} T^{2}} .
$$

Equation (13) may be derived directly from Einstein's expression, $c_{0}=\left\langle(\Delta E)^{2}\right\rangle /\left(k_{B} T^{2}\right)$, since the Gaussian distribution Eq. (12) implies $\left\langle(\Delta E)^{2}\right\rangle=\sigma^{2}$. The equilibrium specific heat increases towards infinity as the temperature goes to zero. While this cannot be the case down to zero temperature in experiment, there is actually a tendency for most supercooled liquids for the excess specific heat to increase as the temperature decreases. ${ }^{2,38}$

The random-walk model was originally proposed by analogy to transport and relaxation of charge excitations in random organic solids, ${ }^{49}$ where the jump rates Eq. (7) are the well-known Miller-Abrahams jump rates for electronic hopping. ${ }^{73}$ There are some differences between the above "derivation" of the random-walk model and Bässler's justification of the model. In Bässler's picture, the experimental dependence of the glass transition temperature on the sample history was understood as an effect due to the density of states depending on the preparation conditions. ${ }^{45}$ In contrast, the above picture is static; the density of states arises from the discretization of the potential energy and does not depend on the conditions of sample preparation. A further difference is that here cooperativity is emphasized, implying $d \gg 1$, while the original Bässler model considered elementary jump processes on a "molecular or weakly cooperative level,"49 implying that $d$ is not much larger than one.

\section{THE ENERGY MASTER EQUATION: AN APPROXIMATION TO THE RANDOM-WALK MODEL}

In order to monitor the average energy during a cooling and subsequent glass transition in the random-walk model, there is no other simple method than to solve the master equation numerically by taking time steps of order $1 / \Gamma_{0}<1$ ps. Clearly, this procedure cannot be used for simulating realistic laboratory time scales of order minutes or hours. In this section an approximation to the random-walk model is derived, which makes it possible to investigate the model on realistic time scales. The approximate equation, termed the "energy master equation," is an equation for the time evolution of the energy probability distribution $P(E, t)$, which ignores the spatial $d$-dimensional structure of the random-walk model.

Consider the random-walk model in many dimensions $(d>>1)$ at low temperatures $\left(k_{B} T<<\sigma\right)$ and long times $\left(t>\tau_{0}\right)$. Whenever $k_{B} T \ll \sigma$ the most likely energies are close to $\bar{E}=-\sigma^{2} \beta<-\sigma$ [Eq. (12)], i.e., deep into the negative tail of the Gaussian. States with these low energies are very rare; nevertheless, at low temperatures the relaxation properties of the random-walk model are dominated by transitions between them. The distance between two low-energy states is large, and a transition between two such states consists of a long and complex path joining neighboring states. It is very hard to calculate the actual transition rate, but it is obvious that the transition rate depends crucially on the maximum energy encountered on the path. Thus, of all possible paths between two low-energy states, the most likely paths are those that have the lowest maximum energy. The value of this maximum energy is identified by percolation theory: ${ }^{70,74-76}$ Imagine the sites of the lattice gradually being filled in order of increasing energy. At a certain filling rate, the site percolation threshold $p_{c}$, the infinite "percolation cluster" of marked sites appears. In two dimensions $p_{c}=0.593$, while in three dimensions $p_{c}=0.312 .{ }^{70}$ In high dimensions one finds ${ }^{76}$ $p_{c} \cong 1 /(2 d-1)$. The highest energy on the percolation cluster, the "percolation energy" $E_{c}$, is given by

$$
\int_{-\infty}^{E_{c}} n(E) d E=p_{c} \text {. }
$$

The percolation energy $E_{c}$ gives a good estimate of the largest energy met on an "optimal" path between two low-energy sites. This is because just above $p_{c}$ a large fraction of the marked sites belongs to the percolation cluster. We thus surmise that the effective transition rate from a low-energy site with energy $E_{i}$ to another low energy site with energy $E_{j}$ is given by the barrier $\Delta E=E_{c}-E_{i}: \quad \Gamma(i \rightarrow j) \propto \exp [-\beta \Delta E]$. This expression satisfies the principle of detailed balance Eq. (6). There are many possible final states, but since each jump rate is given by the above expression, the total rate for jumps away from a site with energy $E, \Gamma(E)$, is given by

$$
\Gamma(E)=\Gamma_{0}^{*} e^{-\beta\left(E_{c}-E\right)} .
$$

To determine $\Gamma_{0}^{*}$ we evaluate $\Gamma\left(E_{c}\right)$ by viewing the percolation cluster as a one-dimensional path, where each site on the average has two neighbors belonging to the cluster. This naive point of view ignores the complicated fractal nature of the cluster, but it does become realistic in high dimensions where it leads ${ }^{76}$ to the correct percolation threshold. Since $E_{c}$ is the largest energy on the percolation cluster, sites with energy $E_{c}$ have on the average two neighbors with lower energy. Thus, the total rate for jumps away from such a site is on the average $2 \Gamma_{0}$, plus some terms for jumps to the higher-energy neighbors. These terms are unimportant at low temperatures, and thus the prefactor of Eq. (15) is given by

$$
\Gamma_{0}^{*}=2 \Gamma_{0}
$$

To arrive at the simplest possible approximate description, the spatial structure of the lattice is now completely ignored. Consequently, all final states are regarded as equally likely, and one arrives at the picture of Fig. 1 which was proposed by Goldstein ${ }^{39}$ in a different context and later discussed in more detail by Brawer. ${ }^{8,43}$

The approximate master equation considers only one variable, the energy. Let $P(E, t)$ denote the energy probability distribution as function of time. Since all final states are regarded as equally likely, the probability of jumping into an energy around $E$ is proportional to the density of states, $n(E)$. The relaxation rate for jumps from states with energy $E$ is $\Gamma(E)$, so the equation for $P(E, t)$ is for some constant $K(t)$ 


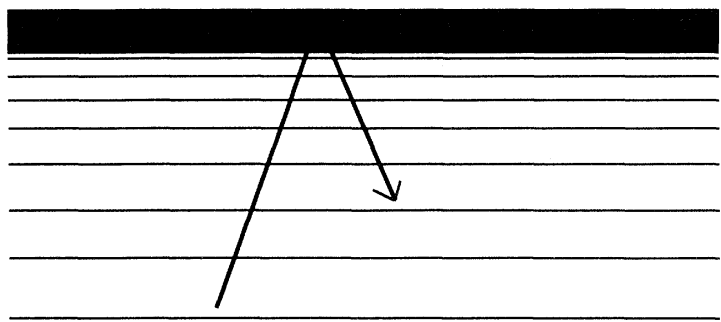

FIG. 1. The Goldstein-Brawer picture of a "flow event" in a viscous liquid (Refs. 8, 39, and 43). The figure illustrates the excitation from one "state," i.e., a potential energy minimum for the molecules in a region of the liquid, to another state - the vertical axis being the energy axis. In Goldstein's model the transition state (black) is identified with the high-temperature, more-fluid, liquid (Ref. 39); Brawer identifies it with a lowdensity state giving room for the molecules to rearrange (Refs. 8 and 43). In the approximate energy master equation (EME) description of Bässler's random-walk model leading to the same picture of a transition, the energy of the transition state is identified with the energy at the percolation threshold [Eq. (14)]. Conversely, the Goldstein-Brawer picture leads to the EME [Eq. (20)] if it is assumed that, once excited into the transition state, the region has forgotten which state it came from and ends up in a randomly chosen state.

$$
\frac{\partial P(E, t)}{\partial t}=-\Gamma(E) P(E, t)+K(t) n(E) .
$$

The constant is determined by requiring conservation of probability:

$$
0=\frac{d}{d t} \int_{-\infty}^{\infty} P\left(E^{\prime}, t\right) d E^{\prime}=\int_{-\infty}^{\infty} \frac{\partial P\left(E^{\prime}, t\right)}{\partial t} d E^{\prime}
$$

implies, since $n(E)$ is normalized, that

$$
K(t)=\int_{-\infty}^{\infty} \Gamma\left(E^{\prime}\right) P\left(E^{\prime}, t\right) d E^{\prime} .
$$

In this reasoning all energies were counted, despite the fact that the picture breaks down for $E>E_{c}$. However, including the energies above $E_{c}$ gives the simplest description and causes little change because high-energy states are very unlikely, anyway. Using Eq. (19), Eq. (17) becomes an integrodifferential equation, ${ }^{44}$ the "energy master equation" (EME),

$$
\begin{aligned}
\frac{\partial P(E, t)}{\partial t}= & -\Gamma(E) P(E, t) \\
& +n(E) \int_{-\infty}^{\infty} \Gamma\left(E^{\prime}\right) P\left(E^{\prime}, t\right) d E^{\prime} .
\end{aligned}
$$

We remind the reader that in this equation the jump rate $\Gamma(E)$ depends on the temperature, which may be an arbitrary function of time.

The EME was first discussed as a model for the thermalization of photoexcited charge carriers in amorphous semiconductors. ${ }^{77,78}$ In this case $n(E)$ is the density of trapping levels in the band gap and $E_{c}$ is the mobility edge of the conduction band. For viscous liquids a similar, but somewhat more complicated master equation was proposed by Brawer in 1984. ${ }^{43}$ Brawer's equation contains an extra entropy factor enumerating the different paths from a particular state to the transition state. A related approach towards relaxation in viscous liquids was advocated by Robertson, Simha, and Curro. ${ }^{79}$ Towards the end of the 1980's Eq. (20) was studied ${ }^{80,81}$ as a model for the relaxation properties of Derrida's random energy model, ${ }^{32}$ and Eq. (20) was proposed as a model for the dynamics of viscous liquids and studied numerically through the glass transition. ${ }^{44}$ Recently, Eq. (20) was used by Arkhipov and Bässler to describe the lowtemperature regime of viscous liquids, assuming that the high-temperature regime is described by the randomwalk model. ${ }^{53}$

The static solution of the EME, $P_{0}(E)$, is given by

$$
P_{0}(E)=\text { const } \frac{n(E)}{\Gamma(E)} \text {. }
$$

This is the canonical probability distribution required by statistical mechanics. In the course of time, the canonical ensemble is realized in a very simple way: All states are visited equally often, but the average time spent in a state with energy $E, 1 / \Gamma(E)$, is proportional to the Boltzmann factor $\exp (-\beta E)$, thus giving the canonical probabilities.

At any fixed temperature an initial nonequilibrium energy probability distribution will approach the equilibrium distribution. This is also the case if the temperature changes in time: At any given time the distribution approaches the equilibrium distribution corresponding to the temperature at that time. Upon continued cooling the system freezes ${ }^{44}$ at the temperature where the time it takes to reach equilibrium becomes larger than the cooling time.

The numerical solution of the EME (detailed in the Appendix) is based on a calculation using the Laplace transformation, ${ }^{82,83}$ resulting in an analytical expression for the relaxation of $P(E, t)$ towards the equilibrium solution $P_{0}(E)$ at a fixed temperature. An arbitrary thermal history is solved by taking small time steps changing the temperature at each step.

\section{COMPUTER SIMULATIONS}

This section reports computer simulations of the random-walk model and compares them to the EME predictions. Results for a continuous cooling and reheating are given, as well as a study of the time evolution of the energy probability distribution for relaxation towards equilibrium at a fixed temperature. Unfortunately, it is impossible to check the validity of the EME description where it is expected to apply best: at low temperatures and long times in many dimensions. This would require enormous computer capacity. All simulations were performed in two dimensions utilizing periodic boundary conditions, and in experiments monitoring relaxation towards equilibrium the lowest temperature studied was $0.25 \sigma / k_{B}$.

A numerical solution of the random-walk model may be obtained by following the motion of a single "particle" in time, the analog of a Monte Carlo simulation. However, this introduces considerable noise and it is much more efficient to solve the master equation Eq. (5) directly. At 
any given time the state of the system is represented by the probabilities, $P_{i}$. In two dimensions any site has four neighbors so the maximum transition rate is $4 \Gamma_{0}$ [Eq. (7)]. In the simulations a time step of length $1 /\left(4 \Gamma_{0}\right)$ was chosen. For each pair of neighboring sites, $A$ and $B$, the new probabilities were found as follows: If $\Delta E=E_{A}-E_{B}>0$, the probabilities are changed according to

$$
\begin{aligned}
& \Delta P_{A}=-\frac{1}{4} P_{A}+\frac{1}{4} e^{-\beta \Delta E} P_{B}, \\
& \Delta P_{B}=-\Delta P_{A} .
\end{aligned}
$$

For one time step each site is upgraded four times according to Eq. (22) (each time, of course, the nonupgraded probabilities from the previous time step are used as $P_{A}$ and $P_{B}$ ). This time-discretization of the master equation is quite crude, but at long times it is sufficiently accurate. The important thing is to ensure exact probability conservation in each time step.

Figure 2 shows the glass transition monitored via the "dynamic" specific heat during a cooling to zero temperature at a constant rate and a subsequent reheating at the same rate. The dynamic specific heat $c$ is defined by
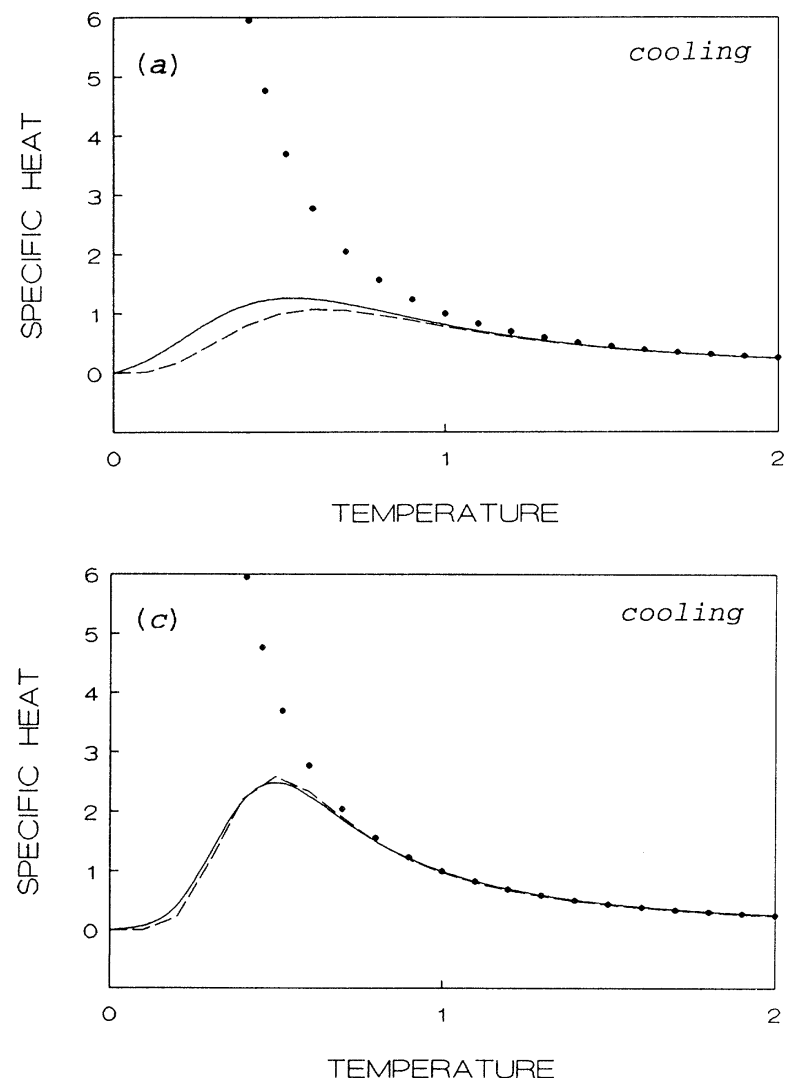

$$
c=\frac{d E / d t}{d T / d t}
$$

The full curves give the results of the simulations of the random-walk model, the dashed curves the EME predictions, and the dots indicate the thermal equilibrium specific heat [Eq. (13)]. Figure 2(a) and 2(b) show $c(t)$ for cooling and reheating from $T=2 \sigma / k_{B}$ to $T=0$ in the time $100 / \Gamma_{0}$, while $2(\mathrm{c})$ and $2(\mathrm{~d})$ show the same but in the time $10000 / \Gamma_{0}$. As expected, the EME predictions work better in the latter case.

Figure 3 shows the frozen-in energy for the cooling, i.e., the energy at zero temperature, as function of the cooling rate. The full curve gives the results of the simulations and the dashed curve the EME prediction.

Figures 4 and 5 show thermalization of the energy probability distribution, starting in equilibrium at one temperature and suddenly changing the temperature. In Fig. 4 the temperature was suddenly lowered. Four snapshots are shown giving the simulation results (full curves) and the EME predictions (dashed curves). The dots indicate the equilibrium energy probability distribution which is approached as $t \rightarrow \infty$. There is a sliding ap-
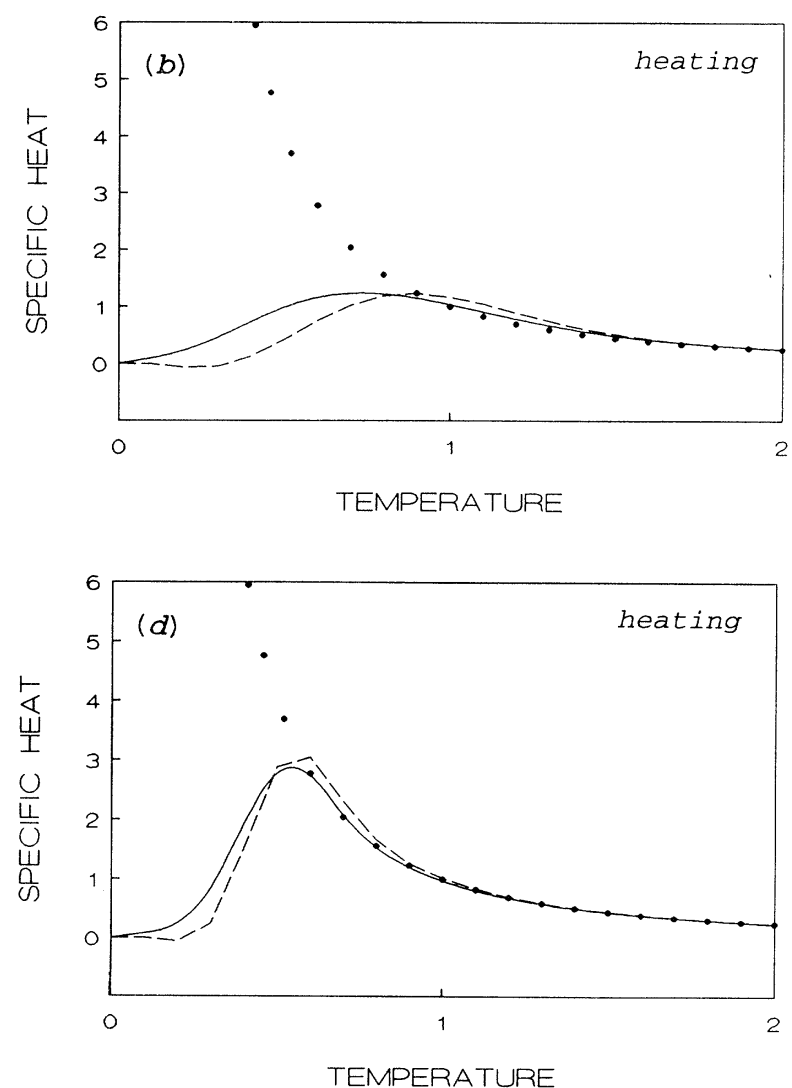

FIG. 2. The glass transition in the random-walk model in two dimensions monitored via the dynamic specific heat (full curves) during a cooling at constant rate to zero temperature and subsequent reheating at the same rate. The dashed curves give the EME predictions, and the dots mark the thermal equilibrium specific heat [Eq. (13)]. (a) shows the specific heat [ $\left.k_{B}\right]$ as function of temperature $T\left[\sigma / k_{B}\right]$ for cooling in the time $t=100\left[1 / \Gamma_{0}\right]$ starting from equilibrium at $T=2.0$; (b) gives the reheating data. (c) and (d) are similar but with cooling and reheating time $t=10000$. The random-walk model data were obtained by averaging ten simulations of a $50 \times 50$ lattice. The EME was solved by the method detailed in the Appendix. Clearly, the EME works better for the slower cooling rate. 


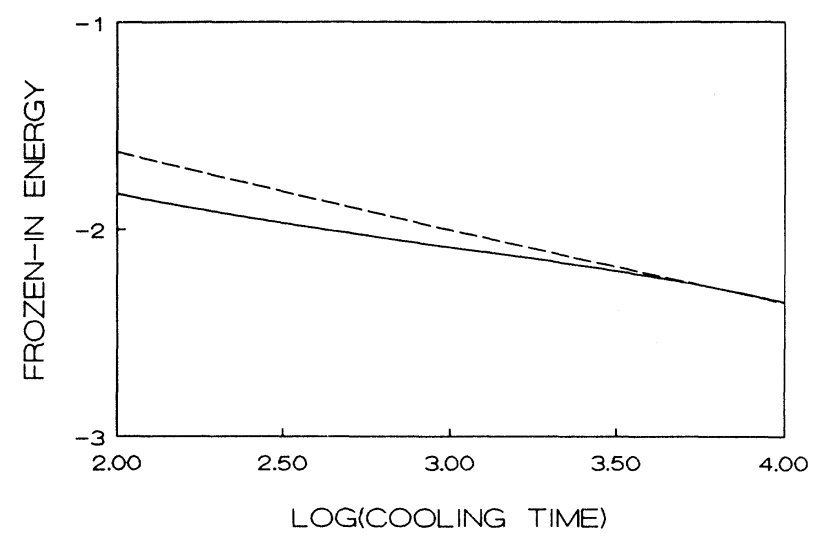

FIG. 3. The average frozen-in energy $[\sigma]$ at zero temperature as function of the logarithm (base 10) of the cooling time $\left[1 / \Gamma_{0}\right]$ for coolings starting from equilibrium at $T=2.0$ $\left[\sigma / k_{B}\right]$. The full curve gives the results based on simulations for each half decade of the random-walk model (each simulation consists of ten averages of a $50 \times 50$ lattice), and the dashed curve gives the EME predictions which are best at long cooling times. The approximate linear relation between the frozen-in energy and the cooling time reflects what is sometimes called "In $(t)$ kinetics" (Refs. 107 and 108).
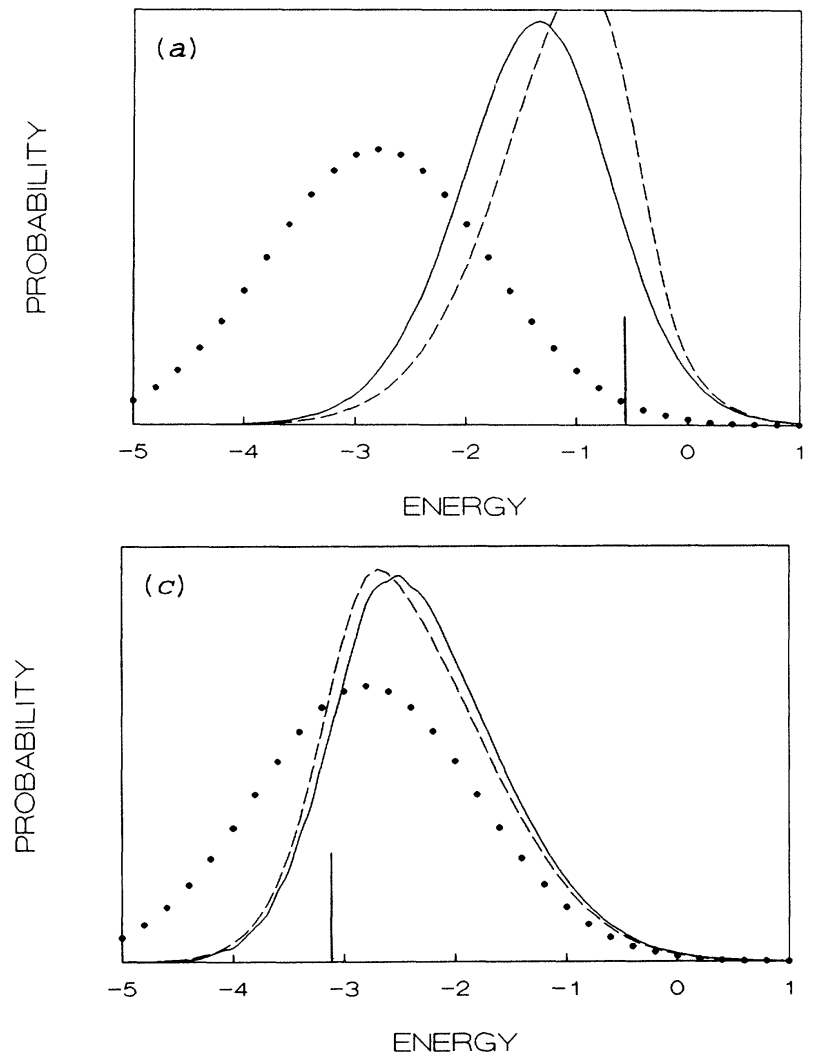

proach towards the equilibrium distribution. This is not the case for a sudden change from a low temperature to a higher temperature (Fig. 5). Here a two-bump distribution occurs (see Sec. VI), a phenomenon that was predicted within the EME. ${ }^{82}$

In Figs. 4 and 5 what happens is that most states with energy $E$ below a characteristic energy $E_{d}$ are frozen, while above $E_{d}$ there is almost thermal equilibrium. The energy $E_{d}$, which is marked by the vertical lines, is the "demarcation energy" that was introduced by Arkhipov et $a l$. in the theory for excited charge-carrier thermalization in amorphous semiconductors. ${ }^{77}$ At any time $t$, $E_{d}(t)$ is found by putting $\Gamma\left(E_{d}\right)$ [Eq. (15)] equal to $1 / t$ (where $T$ is the temperature during the relaxation process):

$$
E_{d}(t)=E_{c}-k_{B} T \ln \left(\Gamma_{0}^{*} t\right) .
$$

\section{THE GLASS TRANSITION ACCORDING TO THE ENERGY MASTER EQUATION}

The previous section showed that the EME gives a good fit to the random-walk model. The glass transition was studied by the EME some time ago, ${ }^{44}$ and this sec-
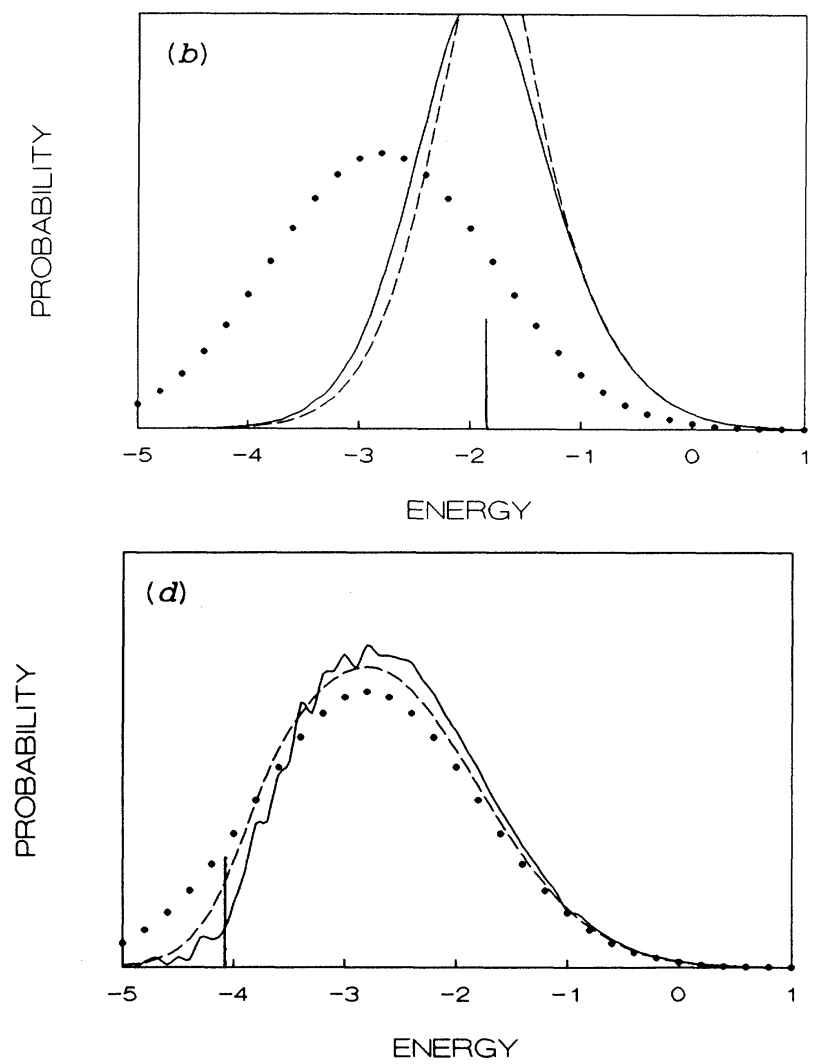

FIG. 4. Relaxation of the energy probability distribution in the random-walk model, $P(E, t)$, towards thermal equilibrium upon a sudden lowering of the temperature starting at equilibrium. The figure shows four snapshots of $P(E, t)$ (full curves) and the EME predictions (dashed curves) starting at $T=2.0\left[\sigma / k_{B}\right]$ lowering the temperature at $t=0$ to $T=0.357$ at the following times $\left[1 / \Gamma_{0}\right]$ : (a) $t=5$, (b) $t=184$, (c) $t=5953$, (d) $t=80752$. The vertical lines mark the demarcation energy $E_{d}$ defined at time $t$ by Eq. (24). In the approximate EME description most states with $E<E_{d}$ have not jumped since $t=0$. As $t \rightarrow \infty, E_{d} \rightarrow-\infty$ and thermal equilibrium is reached. For (a)-(c) the full curves give results for averages of ten simulations of a $1000 \times 1000$ lattice, while for (d) only three simulations were possible for this large lattice (thus, giving rise to more noise). 
tion reviews the findings of Ref. 44 to discuss what to expect at the glass transition in the random-walk model.

In the EME the system is completely characterized by the energy probability distribution $P(E, t)$. At the glass transition temperature $T_{g}, P(E, t)$ freezes and stops changing upon further cooling. Only in some cases is the frozen-in energy distribution equal to the equilibrium energy probability distribution at $T_{g} .{ }^{44}$ To understand this phenomenon it is convenient again to refer to the demarcation energy $E_{d}$, which however now acquires a meaning slightly different from that of Sec. IV: Suppose the liquid is cooled at a constant rate to zero temperature in a time $t_{c}$, starting at equilibrium at some high temperature where the average relaxation time is much smaller than $t_{c}$. At any time during the cooling, the demarcation energy is defined as the energy separating nonfrozen states from the states that are frozen from that time on. If $t_{L}$ is the time left before zero temperature is reached, $E_{d}$ is given by $E_{d}(t)=E_{c}-k_{B} T(t) \ln \left(\Gamma_{0}^{*} t_{L}\right)$. In realistic cases, the glass transition takes place at a $t_{L}$ which is of the same order of magnitude as $t_{c}$ and much larger than $1 / \Gamma_{0}^{*}$. Since $\Gamma_{0}^{*} t_{L}$ in the expression for $E_{d}(t)$ enters only in a logarithm, $t_{L}$ may to a good approximation be replaced by the cooling time $t_{c}$ :

$$
E_{d}(t)=E_{c}-k_{B} T(t) \ln \left(\Gamma_{0}^{*} t_{c}\right) .
$$

Note that $E_{d}(t)$ increases with time during the cooling,
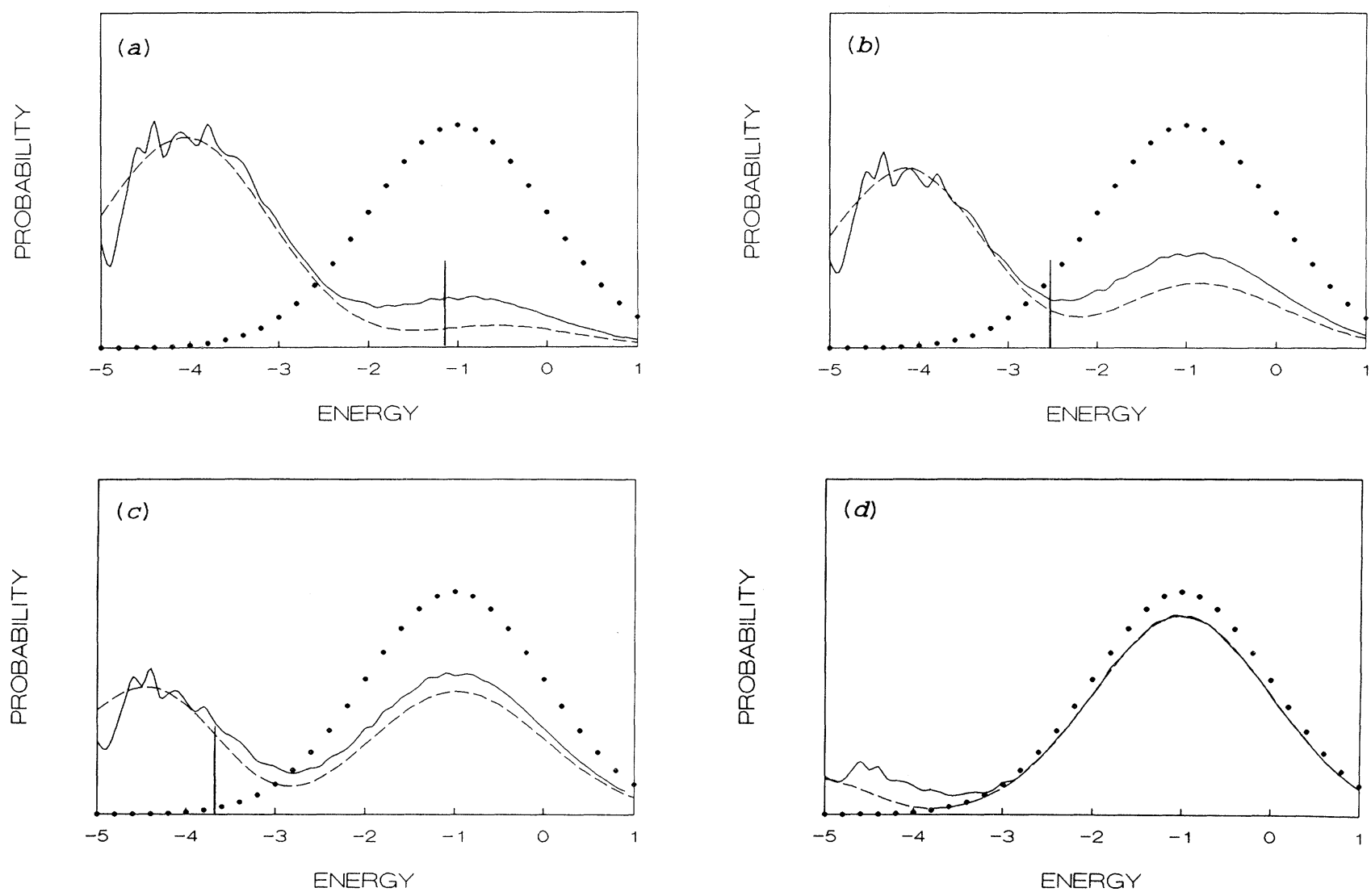

FIG. 5. Relaxation of the energy probability distribution in the random-walk model, $P(E, t)$, towards thermal equilibrium upon a sudden raising of the temperature starting at equilibrium. The figure shows four snapshots of $P(E, t)$ (full curves) and the EME predictions (dashed curves) starting at $T=0.25\left[\sigma / k_{B}\right]$ at time $t=0$ and subsequently annealing at the temperature $T=1.0$. The snapshots are taken at the following times $\left[1 / \Gamma_{0}\right]$ : (a) $t=2$, (b) $t=8$, (c) $t=25$, (d) $t=126$. The vertical lines mark the demarcation energy $E_{d}$ defined at time $t$ by Eq. (24). In the approximate EME description most states with $E<E_{d}$ have not jumped since $t=0$. As $t \rightarrow \infty, E_{d} \rightarrow-\infty$ and thermal equilibrium is reached. The full curves give results for averages of 20 simulations of a $1000 \times 1000$ lattice. (In both Figs. 4 and 5 very large lattices are needed to minimize the statistical fluctuations and to be able to move deep into the Gaussian tail.) The noise seen at low energies is statistical noise due to the fact that there are very few states in the deep Gaussian tail. The two-bump distribution appearing at intermediate times during the annealing reflects that, once a populated state has jumped away from its low energy, it almost immediately thermalizes. This is because there are many high-energy states which are easy to find. Since the region energy must correlate with the volume (because the liquid has a thermal-expansion coefficient which is larger than that of the glass), the model predicts that there is an anomalously large $\mathrm{x}$-ray scattering at intermediate times during the annealing. 
whereas in Sec. IV it decreased with time. In thermal equilibrium the energy probability distribution is a Gaussian centered around $\bar{E}(T)$ [Eq. (12)]. As the temperature is lowered, the equilibrium Gaussian is displaced towards lower energies while at the same time $E_{d}(t)$ increases. When the Gaussian meets $E_{d}(t)$, the glass transition takes place. ${ }^{44}$ This happens when $\bar{E}[T(t)]=E_{d}(t)$. For the system with constant specific heat $c k_{B}$ studied in Ref. 44, corresponding to $n(E) \propto E^{c-1}, 0<E<E_{c}$ (where $E_{c}$ now plays the additional role of a cutoff), one has at low temperatures $\bar{E}(T)=c k_{B} T$. From $\bar{E}=E_{d}$ one finds that the glass transition temperature is given by

$$
k_{B} T_{g}=\frac{E_{c}}{c+\ln \left(\Gamma_{0}^{*} t_{c}\right)} .
$$

A linear relationship between $1 / T_{g}$ and the logarithm of the cooling time is often observed in experiment. ${ }^{84}$

For the freezing of the energy probability distribution there are two different limiting scenarios, depending on the rate of change with temperature of $E_{d}$ and $\bar{E}$, respectively. In the model studied in Ref. 44, $d \bar{E} / d T=c k_{B}$ and $d E_{d} / d T=-k_{B} \ln \left(\Gamma_{0}^{*} t_{c}\right)$. The case when $E_{d}$ changes much faster with temperature than $\bar{E}$ was referred to as a "slow" glass transition, since it requires long cooling times: $\ln \left(\Gamma_{0}^{*} t_{c}\right) \gg c$. In this case the equilibrium Gaussian almost does not move at all when the demarcation energy passes it and freezes in the energies. Thus, the frozen-in energy distribution, $P_{f}(E)$, is close to that corresponding to thermal equilibrium at $T=T_{g}$ :

$$
P_{f}(E) \cong \frac{1}{\sqrt{2 \pi\left\langle(\Delta E)^{2}\right\rangle}} \exp \left[-\frac{\left(E-E_{g}\right)^{2}}{2\left\langle(\Delta E)^{2}\right\rangle}\right],
$$

where $E_{g}=c k_{B} T_{g}$ and $\left\langle(\Delta E)^{2}\right\rangle=c\left(k_{B} T_{g}\right)^{2}$. The other limiting case is that of a "fast" glass transition: $\ln \left(\Gamma_{0}^{*} t_{c}\right)<<c$. Here, the demarcation energy moves very slowly compared to the Gaussian and is almost constant during the glass transition. To determine $P_{f}(E)$ consider the energy fluctuations of a single region. As long as its energy is above the demarcation energy, the region "jumps" many times between the high-energy common states. Sooner or later, however, the region ends up in a state below $E_{d}$, or just above $E_{d}$, being subsequently frozen when $E_{d}$ passes. As for all other jumps, this last jump hits an energy with a probability proportional to the density of states. Around $E_{g}$ the density of states is proportional to $\exp \left[E /\left(k_{B} T_{g}\right)\right],{ }^{72}$ so the normalized $P_{f}(E)$ is roughly given by (where again $E_{g}=c k_{B} T_{g}$ )

$$
P_{f}(E) \cong\left\{\begin{array}{l}
\frac{1}{k_{B} T_{g}} \exp \left[\frac{\left(E-E_{g}\right)}{k_{B} T_{g}}\right], \quad E<E_{g} \\
0, \quad E>E_{g} .
\end{array}\right.
$$

In Ref. 44 the predicted exponential increase of $P_{f}(E)$ below $E_{g}$ was confirmed in the numerical solution of the master equation; above $E_{g}$, however, $P_{f}(E)$ did not drop discontinuously to zero, but followed a Gaussian decay.

The conclusion from the above is that, in general, one cannot expect a glass merely to have the structure of the equilibrium liquid at $T=T_{g}$. Certainly, the average frozen-in energy is equal to the average energy of the equilibrium liquid at $T_{g}$, but the distribution of frozen-in energies in the glass may be different from that corresponding to the equilibrium liquid. This has consequences for the glass properties. In the glass, any physical property which is a function of the region energy (if it depends linearly on $E$ for the relevant energies) is, depending on the cooling rate, distributed according to a Gaussian or an exponential. Along these lines it has been argued that amorphous semiconductors prepared by a fast glass transition have exponential band tails. ${ }^{85}$

It is convenient to define a number that distinguishes between the two types of glass transitions. This number, denoted by $\iota$, is the absolute value of the ratio between the change in the average energy and the change in the demarcation energy at the glass transition:

$$
\iota=\left|\frac{d \bar{E}}{d E_{d}}\right|\left(T_{g}\right) .
$$

"Slow" glass transitions arise whenever $\iota \ll<$, while "fast" glass transitions correspond to $\iota \gg>1$.

We now proceed to calculate the $\iota$ parameter for the random-walk model from the approximate EME description. The average energy is given by Eq. (12), $\bar{E}=-\sigma^{2} /\left(k_{B} T\right)$. Thus, the equation determining $T_{g}$, $\bar{E}=E_{d}$ is

$$
-\sigma^{2} /\left(k_{B} T_{g}\right)=E_{c}-k_{B} T_{g} \ln \left(\Gamma_{0}^{*} t_{c}\right),
$$

or

$$
\ln \left(\Gamma_{0}^{*} t_{c}\right)\left(k_{B} T_{g}\right)^{2}-E_{c}\left(k_{B} T_{g}\right)-\sigma^{2}=0 .
$$

The positive solution of this equation is

$$
k_{B} T_{g}=\frac{E_{c}+\sqrt{E_{c}^{2}+4 \sigma^{2} \ln \left(\Gamma_{0}^{*} t_{c}\right)}}{2 \ln \left(\Gamma_{0}^{*} t_{c}\right)} .
$$

Since $d \bar{E} / d T=\sigma^{2} /\left(k_{B} T^{2}\right)$ and $d E_{d} / d T=-k_{B} \ln \left(\Gamma_{0}^{*} t_{c}\right)$ the $\iota$ parameter is via Eqs. (29) and (30) given by

$$
\begin{aligned}
\iota & =\frac{\sigma^{2}}{\left(k_{B} T_{g}\right)^{2} \ln \left(\Gamma_{0}^{*} t_{c}\right)} \\
& =1-\frac{E_{c}}{\left(k_{B} T_{g}\right) \ln \left(\Gamma_{0}^{*} t_{c}\right)} .
\end{aligned}
$$

If the dimension $d>2$, the percolation energy $E_{c}$ is negative and Eq. (31) implies

$$
d>2: \frac{E_{c}}{k_{B} T_{g}}=-\frac{2 \ln \left(\Gamma_{0}^{*} t_{c}\right)}{\sqrt{1+4\left(\sigma^{2} / E_{c}^{2}\right) \ln \left(\Gamma_{0}^{*} t_{c}\right)}-1} .
$$

When Eq. (33) is substituted into Eq. (32) one gets

$$
d>2: \quad \iota=1+\frac{2}{\sqrt{1+4\left(\sigma^{2} / E_{c}^{2}\right) \ln \left(\Gamma_{0}^{*} t_{c}\right)}-1} .
$$

In the case $d=2, E_{c}$ is positive and, as is easy to show, the $\iota$ parameter is given by

$$
d=2: \quad \iota=1-\frac{2}{\sqrt{1+4\left(\sigma^{2} / E_{c}^{2}\right) \ln \left(\Gamma_{0}^{*} t_{c}\right)}+1} .
$$




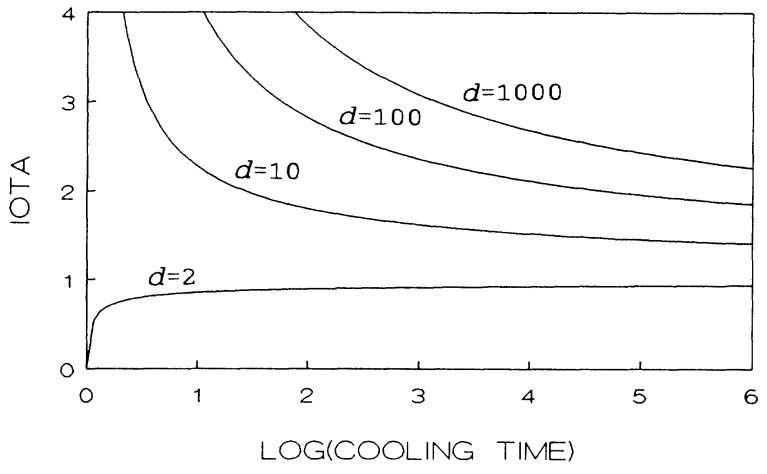

FIG. 6. The parameter $\iota$ [Eqs. (29), (34), and (35)] characterizing the glass transition in the random-walk model for different dimensions $(d=2,10,100,1000)$ as function of cooling time $\left[1 / \Gamma_{0}^{*}\right]$ according to the EME. The difference between $d=2$ and $d>2$ arises from the fact that only in two dimensions is the percolation energy positive. If $\iota \ll<$ the transition is a "simple freezing" glass transition, where the energy probability distribution of the glass is the equilibrium distribution at $T_{g}$, frozen-in almost unmodified. In the other limit, $\iota \gg 1$, the transition is a "relaxational" glass transition, where relaxations at the glass transition considerably deform the equilibrium energy probability. As a result, the glass does not acquire a structure corresponding to the equilibrium liquid at $T_{g}$. For very long cooling times one ends up in the mixed case $\iota=1$ where there is some relaxation at $T_{g}$.

Figure 6 gives the $\iota$ parameter as function of the cooling time for $d=2,10,100,1000$.

The terminology of Ref. 44 referring to "slow" and "fast" glass transitions is not appropriate for the random-walk model. For this model, as the cooling time goes to infinity, one finds $\iota \rightarrow 1$ in all dimensions (Fig. 6); thus there are no "slow" glass transitions for slow cooling rates. On the other hand, whenever $d>2$ the glass transition is "fast" for sufficiently small cooling times; the case $d=2$ is peculiar in that the glass transition is "slow" for fast coolings. In view of this it is better to refer to glass transitions with $\iota \gg 1$ [previously: "fast"] as "relaxational" glass transitions: These are the interesting cases where relaxation processes right at the glass transition result in a frozen-in energy distribution different from the equilibrium distribution at $T_{g}$. The cases when $\iota<<1$ [previously: "slow"] may be referred to as "simple freezing" glass transitions; here the equilibrium energy distribution is simply frozen-in at $T_{g}$.

\section{DISCUSSION}

In this paper Bässler's random-walk model for viscous liquids and the glass transition was "derived" in Sec. II. In Secs. III and IV, it was argued physically and illustrated by computer simulations that the energy master equation (EME) gives a good fit to the random-walk model. Thereby, two at first sight quite different approaches to the glass transition problem ${ }^{44,45}$ are unified. The EME is an equation for the energy probability distribution, which even for an arbitrarily varying temperature and for very long times makes it possible to calculate this quantity. However, it should be remembered that the EME only deals with energy; there a number of interesting properties of the random-walk model that relate to the displacement of the "particle," which are not dealt with by the EME.

The below discussion is sectioned into eight parts, the first four (A)-(D) discuss the random-walk model and its connection to the EME, while the last four $(\mathrm{E})-(\mathrm{H})$ deal with the EME itself as a model for viscous liquids and the glass transition.

\section{A. The random-walk model in the present paper}

The physical justification of the random energy model was discussed in detail in Sec. II. The most drastic approximation $^{29}$ is the partitioning of the liquid into noninteracting regions, an approximation that must be made to arrive at a tractable model. Replacing the deterministic equations of classical physics with stochastic equations seems more acceptable, though not without pitfalls. ${ }^{67,86}$ A further approximation is the replacing of "complexity" by "randomness."69 This, in conjunction with the discretization of state space lead to the model of a random walk on a lattice with random energies. Models involving random walks in random environments ("rugged" energy landscapes) have been used in many contexts. ${ }^{48,70,75,86-91}$ In formulating a model of this type one is led to ask whether the energy minima or the energy maxima should vary randomly, or both. The random-walk model gives a simple and beautiful answer to this question: No states are appointed "maxima" or "minima." All states are equal, but the higher-energy states behave as maxima, being part of the paths between the populated, but rare, low-energy states. The assumption of Gaussianly random energies is the simplest choice. Fortunately, it leads to an equilibrium specific heat which increases with decreasing temperature, as seen in experiment.

Following the ideas of Derrida's "random energy model" 32 it is possible to discuss the Kauzmann paradox within the model: Since there is only a finite "excess" entropy of the supercooled liquid, a truncation of the Gaussian at some low energy is forced on the model to avoid the Kauzmann paradox. ${ }^{92,93}$ This truncation implies that the thermodynamics of the model becomes almost indistinguishable from that of Derrida's "random energy model." The truncation has not been discussed here, because the truncated random-walk model does not reproduce the experimental correlation between the Kauzmann temperature and the $T_{0}$ of the VFT law Eq. (2).

The random-walk model contains three parameters. There are two scaling parameters, the width of the Gaussian $\sigma$ and the attempt frequency $\Gamma_{0}$, while the third parameter is the dimensionless state space dimension $d$. For a qualitative discussion of the model there is thus only one relevant parameter $d$. 


\section{B. A comparison to the original approach of Bässler and co-workers}

Bässler and co-workers ${ }^{45,46}$ justified the Gaussian density of states by reference to the central-limit theorem, assuming that the region energy is a sum of a large number of independent contributions. However, one might similarly argue that any macroscopic system has a Gaussian density of states, implying that any such system has a specific heat varying with temperature as $T^{-2}$, which is clearly incorrect. In the present paper the Gaussian is an ad hoc assumption, only justified from the fact that it gives a "thermodynamic" density of states [Eq. (11)]. A further difference is that Bässler and co-workers assumed that the density of states fluctuates in time. This justified their use of Metropolis dynamics, since the "particle" awaits a favorable time for jumping where the barrier to be overcome is negligible. Here, the density of states is assumed constant and time independent. This difference in the two approaches means that the present work cannot maintain the original interpretation of the fact that $T_{g}$ depends on sample history. This was explained ${ }^{45}$ as a logical consequence of the fact that the density of states depends on preparation conditions. However, even for a constant density of states does the glass transition temperature depend on sample history.

The most elaborate version of the Bässler model was given in a recent paper by Arkhipov and Bässler. ${ }^{53}$ They distinguish between a high-temperature regime described by the random-walk model and a low-temperature regime described by the EME. The present work fully confirms this picture. Here, however, the random-walk model is assumed to be the underlying model at all temperatures, and the parameters of the low-temperature approximate EME are uniquely determined from the random-walk model.

\section{From the random-walk model to the EME}

At low temperatures the populated states of the random-walk model are rare low-energy states, and the transitions between these far apart states follow the optimal paths, the ones that have the lowest maximum energy. The distance between two low-energy states is large and the maximum energy of an optimal path is close to the percolation energy defined from the site percolation threshold by Eq. (14). A number of authors have previously emphasized the importance of percolation at the glass transition, ${ }^{13,40,94-96}$ but in contexts different from the present.

The importance of percolation at low temperatures means that the random-walk model here is regarded as consisting of states (= the deep minima) separated by barriers of the same height. Effectively, the model reduces to a model of the "trapping" type used, e.g., for describing trapping of electrons in amorphous semiconductors. Interestingly, it has previously been noted that the predictions of trapping models are almost indistinguishable from the predictions of the EME. ${ }^{97,98}$

The existence of the percolation energy makes it possible to distinguish two temperature regimes for the random-walk model, a high-temperature regime opposed to the low-temperature regime where $|\bar{E}(T)| \gg\left|E_{c}\right|$. In the high-temperature regime, the most likely states usually have one or more neighbors with a lower energy and these states consequently have a very short "lifetime." In the low-temperature regime, typical populated states are surrounded by states all of which have a higher energy. Only well into the low-temperature regime does the approximate EME description apply. This picture, which is valid whenever $d>2$ (for $d=2$ there is no hightemperature regime), is close to that recently advocated in general terms by Hunt. ${ }^{13,95} \mathrm{He}$ predicts that viscous liquids have a high-temperature regime described by effective-medium type theories and a low-temperature regime where percolation effects dominate. A two regime picture also results from the mode-coupling theory, ${ }^{26}$ but in a different context.

The low-energy states could be thought of as effectively including a number of their relatively low-energy neighboring states, thus forming rather complex low energy "basins", in agreement with the ideas of Stillinger and Weber $^{63,64}$ and Angell. ${ }^{11,30}$ The complexity of the basins implies that considerable entropy resides inside each basin. ${ }^{11,99}$ Note that this picture of complex minima derives from a model where neighboring energies are completely uncorrelated.

A transition between two low-energy states is a complex sequence of steps. Such a transition involves an element of cooperativity ${ }^{8,29}$ in the sense that a long sequence of jumps is undertaken in order to have a successful transition. Thus, at low temperatures the random-walk model contains both cooperativity and heterogeneity, the two factors identified by Scherer $^{29}$ as being important for any realistic model of viscous liquids. The random-walk model also conforms to the thoughts of Goldstein in 1969, expressing a firm belief that, "when all is said and done, the existence of potential energy barriers large compared to the thermal energy are intrinsic to the occurrence of the glassy state, and dominate flow, at least at low temperatures." 42

The approximate EME description of the random-walk model ignores the spatial structure of the state space (the only trace left being the $d$ dependence of the percolation energy). In the limit of large $d$ this is not unrealistic, since there are many deep energy minima available not too far from a given minimum. Consequently, transitions to all states should be allowed with equal probability, as in the EME.

\section{Computer simulations: Comparing the random-walk model to the EME predictions}

The approximate EME description makes it possible to study the random-walk model for realistic long times. This involves a numerical implementation of the analytic EME solution valid for the approach to thermal equilibrium at a fixed temperature (Appendix). In order to check the validity of the EME approximation, computer simulations were carried out (Sec. IV). The EME is expected only to be valid in many dimensions at low temperatures and long times, a regime that cannot be studied by even the fastest computers available today because of two problems: At low temperatures the most likely states are 
very rare so enormous lattices are needed; also, the relaxation times are extremely long. Instead, the simulations were carried out in two dimensions and at moderate temperatures. Despite this, the computer simulations revealed a rather good agreement with the EME predictions. A numerical study of thermalization in the random-walk model was previously performed by Bässler and co-workers, starting in equilibrium at infinite temperature. $^{100}$ In Figs. 4 and 5 of the present paper, the thermalization was studied going from one finite to another finite temperature. A surprising thing happens in the more exotic case going from a low to a high temperature (Fig. 5) where a two-bump structure appears at intermediate times, a phenomenon that is reproduced by the EME. Thus, if the random-walk model is realistic, one may induce a "dynamically generated phase separation" in a glass by the following procedure: Anneal the glass for a very long time at a relatively low temperature, then increase the temperature and finally quench the glass at the right time in order to catch it in a state corresponding to Fig. 5(c). The dynamically generated phase separation results in a glass consisting of low-energy regions and high-energy regions, but only few of intermediate energy. Such a glass has a well-defined correlation length, equal to the region size.

The rest of Sec. VI deals with the EME independently of its connection to the random-walk model.

\section{E. The EME as the simplest possible truly cooperative master equation, "derived" \\ from the non-Arrhenius temperature dependence of the average relaxation time}

Since most naive phenomenological models involving a distribution of energy barriers give an average relaxation time $\tau(T)$ with an apparent activation energy that decreases with decreasing temperature, the observed nonArrhenius $\tau(T)$ must contain an important clue to the construction of a phenomenological model. Assuming that the activation entropy plays little role we write

$$
\tau(T)=\tau_{0} e^{\Delta E(T) /\left(k_{B} T\right)} .
$$

Experiments imply that $\Delta E(T)$ increases as the temperature decreases. The simplest way to explain this is as follows: $\Delta E(T)$ is the difference between the barrier to be overcome and the most likely region energy. If a region contains many molecules ("cooperativity"), the most likely energy is by general thermodynamic principles close to the average energy $\bar{E}(T)$. If furthermore the maximum to be overcome is assumed to be constant, $=E_{c}$, one has

$$
\Delta E(T)=E_{c}-\bar{E}(T)
$$

Since $\bar{E}(T)$ decreases with decreasing temperature, the barrier increases. This simple idea is the basis for the EME as a model for viscous liquids (independent of the random-walk model): Equation (37) motivates Eq. (15) and to derive the EME one just needs the further assumption - again the simplest possible - that, once excited into the transition state, a region ends up in a randomly chosen other state. This assumption means that an excitation must be a complete reordering of the region molecules. Thus, the EME is truly cooperative.

\section{F. The EME as the simplest master equation conforming to the Goldstein-Brawer picture (Fig. 1)}

In an interesting paper from 1972 , Goldstein ${ }^{39}$ proposed a picture of viscous flow where the transition state is the "high-temperature, more-fluid, liquid usually studied by theorists." Once excited into this common transition state-being totally different from the potential energy minimum which the region was excited from - the only reasonable assumption is that any other (low energy) state can be reached. In the EME these other states are reached with equal probability. Thus, from Goldstein's ideas $^{39,42}$ one is led almost automatically to the EME. However, Goldstein did not discuss any master equation; a master equation in the spirit of his ideas was set up by Brawer in 1984. ${ }^{8,43}$ Brawer's model is more detailed than Goldstein's and his master equation is more complex than the EME. In the 1985 version of Brawer's model ${ }^{8}$ a region has $K$ volume elements, each of which has two states: a low-density (high-energy) state and a highdensity (low-energy) state. If a certain fraction of the $K$ volume elements are excited into the low-density state, a transition is allowed. The jump thus involves a number of the volume elements forming a complex sequence of density changes, somewhat like a transition between two low-energy states in the random-walk model.

\section{G. The EME interpretation versus the naive interpretation of the activation energy}

Figure 7 sketches typical experimental results for the average relaxation time using an Arrhenius plot (full curves): There is a non-Arrhenius high-temperature regime for the equilibrium viscous liquid and an Arrhenius low-temperature regime (the glass). The naive interpretation of this [Fig. 7(a)] is based on writing $\tau(T)=\tau_{0} \exp \left[\Delta F(T) /\left(k_{B} T\right)\right]$ and using the standard thermodynamic relations $\Delta F=\Delta E-T \Delta S$ and $d \Delta F / d T$ $=-\Delta S$; from this it is easy to show that the activation energy $\Delta E$ is the slope of the tangent (dashed line). This slope changes abruptly at the glass transition, which is sometimes explained as being due to the fact that below $T_{g}$ relaxation takes place in an essentially fixed structure, while above $T_{g}$ the activation energy has an additional contribution from structural changes. Figure $7(\mathrm{~b})$ gives the EME interpretation of data which follows from Eq. (15) [or Eqs. (36) and (37)]. Here, the activation energy $\Delta E$ is the slope of the secant from $\tau(T)$ to $\tau_{0}$. Thus, at $T=T_{g}$ the activation energy simply stops changing, because glassy relaxation takes place in an essentially fixed structure.

\section{H. A qualitative comparison of the EME to experiments}

The random-walk model has been quantitatively successfully compared to experiments on a number of glassforming liquids. ${ }^{45,46,53}$ We here proceed to argue that the 


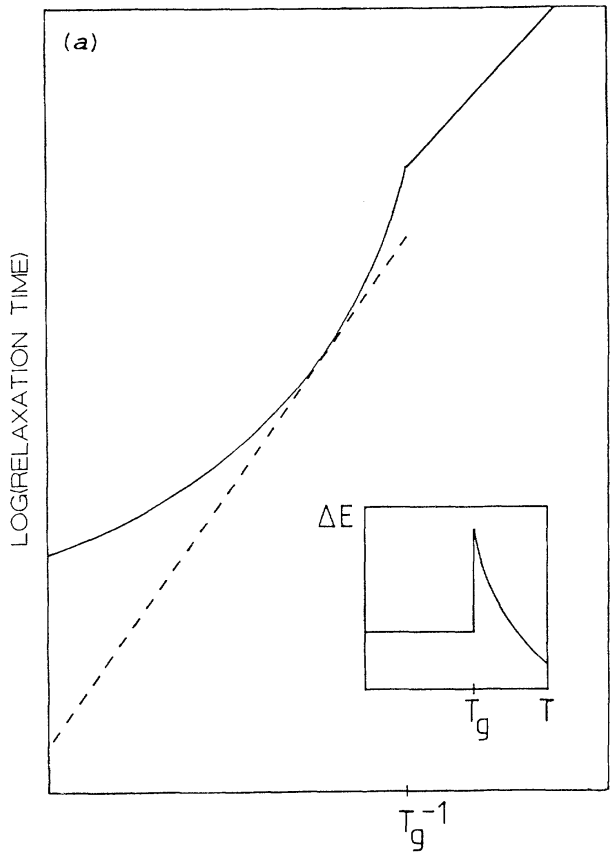

INVERSE TEMPERATURE

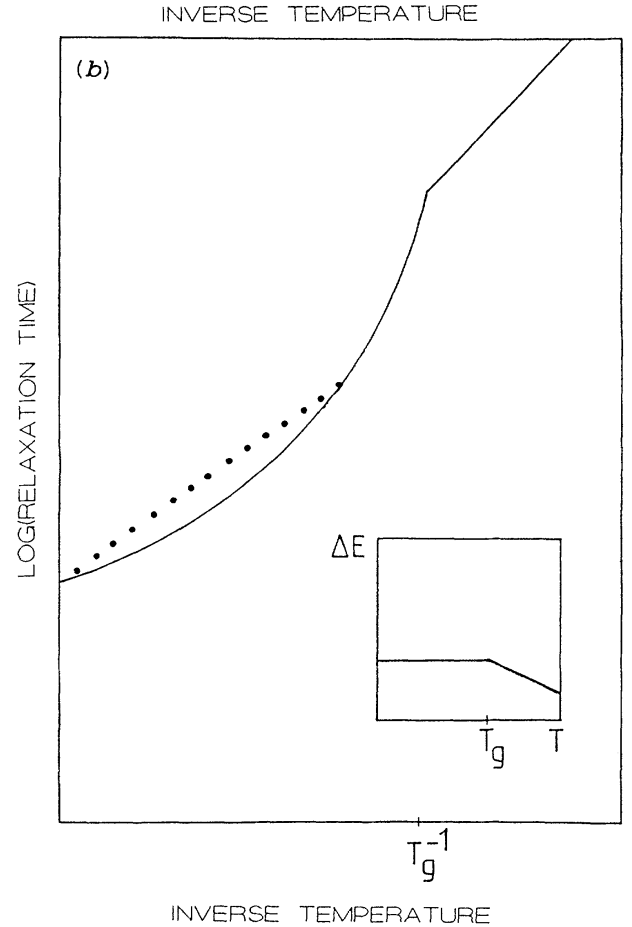

FIG. 7. Naive interpretation of the activation energy (a) compared to the interpretation underlying the EME (b). Both figures show an Arrhenius plot of the same typical average relaxation time data for the supercooled liquid (non-Arrhenius part, $T>T_{g}$ ) and for the glass (Arrhenius part, $T<T_{g}$ ). In (a) the activation energy is interpreted as the slope of the tangent, which changes discontinuously at $T_{g}$. In (b) the activation energy is interpreted as the slope of the secant drawn to the microscopic time. In both cases one finds that the activation energy increases as the temperature decreases. In (b), at the glass transition the activation energy stops increasing and becomes constant. In the naive interpretation (a), the activation energy changes discontinuously at the glass transition.
EME itself, despite being very simple, qualitatively reproduces a large number of experimental observations, yields some new predictions, and also some "wrong" predictions. Most of the properties of the EME listed below will not be detailed here, but are straightforward to derive. ${ }^{82}$

\section{Qualitatively correct predictions of the EME}

(a) The EME gives a qualitatively correct temperature dependence of the average relaxation time above and below $T_{g}$ (Fig. 7);

(b) The preexponential of $\tau(T)$ for glassy relaxation is predicted to be close to $\tau_{0}$, i.e., a phonon time; ${ }^{8,43}$

(c) A true Arrhenius behavior of $\tau(T)$ implies a zero region specific heat and thus no distribution of relaxation times. ${ }^{7,51,101}$ If the region size is universal, as conjectured by Nemilov, ${ }^{102,103}$ there is a correlation between the magnitude of the "excess" specific heat (the configurational specific heat), the degree of nonArrhenius behavior of $\tau(T)$, and the relaxation time distribution width; $7,104,105$

(d) If the region specific heat is regarded as roughly constant close to $T_{g}$, the EME predicts a proportionality between $1 / T_{g}$ and the logarithm of the cooling time; ${ }^{84,106}$

(e) In the glassy state, energy relaxation proceeds according to the EME with a logarithmic time dependence [compare Eq. (24)], " $\ln (t)$ kinetics," $81,107,108$ with a slower than logarithmic time dependence at both the initial and final stages. The logarithmic relaxation law is conventionally explained as being due to a "relaxation" of the relaxation rate itself. $8,29,43,81,82,109$ The EME conforms to this picture in a particularly simple way;

(f) For relaxation upon a sudden change in temperature an asymmetry is predicted between the two possible cases, a well-known phenomenon referred to as "nonlinearity"; ;,29

(g) If one assumes a correlation between the region energy and its volume (which is necessary because the viscous liquid has a larger thermal-expansion coefficient than the glass or crystal), the EME also gives predictions regarding the pressure dependence of the average relaxation time. Writing $\tau(p) \propto \exp [p \Delta V(p)]$, experiments imply that the activation volume increases as the pressure increases. ${ }^{110}$ If $\Delta V(p)=V_{c}-\bar{V}(p)$ just as for the activation energy this observation is explained, since the region average volume decreases with increasing pressure. A further possibility is to assume a linear relation between region volume and energy. In that case the normalized frequency-dependent isothermal compressibility is equal to the normalized frequency-dependent specific heat, as proposed by $\mathrm{Zwanzig}{ }^{111}$ there are some indications that this is the case in experiment. ${ }^{112}$ Also, for quantities uncorrelated with the region energy, it can be shown that the EME predicts a slight "decoupling" of their average relaxation time from that of the frequency-dependent specific heat, where the average relaxation time for the latter becomes somewhat larger (Fig. 8). This is also the case experimentally. ${ }^{112}$ 


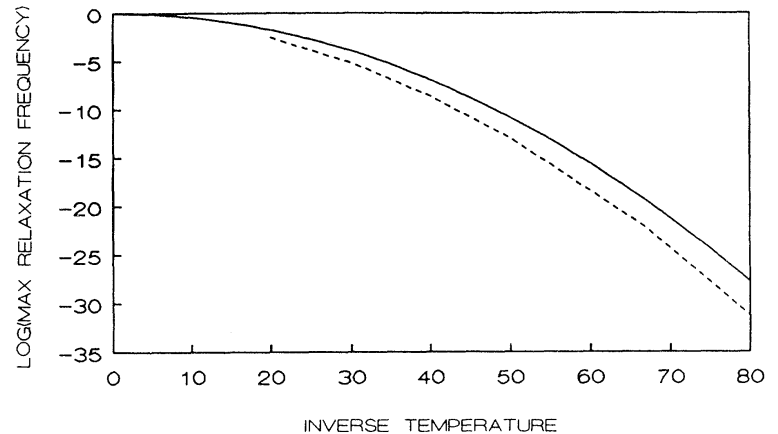

FIG. 8. Decoupling of thermal relaxation times from other relaxation times according to the EME for a Gaussian density of states with $E_{c}=0$. The maximum relaxation frequency is given in units of $\Gamma_{0}^{*}$ and the temperature in units of $\sigma / k_{B}$. The full curve gives the loss peak frequency for a quantity that is uncorrelated to the energy (calculated from its time autocorrelation function). The dashed curve is the specific-heat loss peak frequency (Ref. 117). The figure shows that there is a slight slowing down of thermal relaxations compared to other relaxations, an effect that has been seen in experiments (Ref. 112).

\section{New predictions of the EME}

(a) At low temperatures the average relaxation time of the equilibrium viscous liquid is predicted to become Arrhenius with a pre-exponential equal to $\tau_{0}$. Thus, a change in sign of the curvature of the Arrhenius-plot, $d^{2} \ln [\tau(T)] / d\left(T^{-1}\right)^{2}$, is predicted. Similarly, a change in sign of $d^{2} \ln [\tau(p)] / d p^{2}$ at large pressure is predicted. This follows from the fact that a region must have a lowest energy state or a minimum volume;

(b) The EME gives detailed predictions regarding the nature of the asymmetry of relaxation upon sudden changes in temperature: For a sudden cooling from thermal equilibrium relaxation is predicted to proceed continuously (Fig. 4), while relaxation upon a sudden increase in temperature is peculiar, resulting in a two-bump energy probability distribution at intermediate times (Fig. 5). In the latter case, if the relaxation is interrupted by quenching to low temperatures, one ends up with a strange glass in which some regions have low energy and some have high energy, a "dynamically generated phase separation." The energy correlates with the volume [compare $\mathrm{H} 1 \mathrm{~g}$ ]. Therefore, a dynamically generated phase separated glass will give an anomalous $x$-ray scattering signal. Nemilov has predicted a similar phenomenon on purely thermodynamic grounds; ${ }^{113}$

(c) The EME predicts that there are two different limiting cases of the glass transition (Sec. V), "relaxational" (previously called "fast") glass transitions and "simple freezing" (previously called "slow") glass transitions. The latter type freezes-in the region energy probability distribution at $T_{g}$ and the glass simply inherits the structure of the equilibrium liquid at this temperature. At a relaxational glass transition, relaxation processes right at $T_{g}$ result in a frozen-in region energy distribution different from the equilibrium distribution. (Of course, glasses may be produced by a third kind of process, a quench to low temperatures in a time much shorter than the average relaxation time at the starting temperature - this process clearly results in a frozen-in region energy distribution that is equal to the equilibrium distribution at the starting temperature.)

\section{3. "Wrong" predictions of the EME}

(a) The VFT law Eq. (2) is inconsistent with the EME which predicts a finite average relaxation time at all temperatures. Experimentally, however, deviations from this law seem to occur for large viscosities, where the data exhibit a less dramatic temperature-dependence than predicted; ${ }^{20,29,51}$

(b) The Kauzmann paradox is also inconsistent with the EME (without a low-energy cutoff in the density of states), which at all temperatures predicts a positive specific heat. However, a suitably chosen region density of states (e.g., a truncated Gaussian ${ }^{32,92,93}$ ) easily reproduces the experimental configurational entropy;

(c) The Kohlrausch-Williams-Watts law (stretched exponentials) for the time dependence of the energy relaxation is not reproduced by the model. However, the EME does predict broad distributions of relaxation times.

These three points are places where the EME on the one hand does not reproduce the conventional picture of viscous liquids and glassy relaxation, but on the other hand is not inconsistent with experiment. The final point to be mentioned here is a more serious objection to the EME:

(d) If the correct non-Arrhenius behavior of $\tau(T)$ is to be reproduced by choosing a suitable $n(E)$ (possibly a non-Gaussian), the model predicts a peak of the imaginary part of the frequency-dependent linear specific heat $^{114}$ that is too broad. This conclusion seems to hold, despite the fact that only few measurements of this quantity have been published and that there is a considerable discrepancy between the results of Christensen ${ }^{115}$ and those of Birge and Nagel. ${ }^{116}$ This disagreement between the EME and experiment means that the EME is too simple to be realistic. Preliminary work ${ }^{117}$ indicates that it is possible to solve this problem and still retain the region assumption and the assumption that the only important parameter is the region energy. This is done by the following extension of the EME. One introduces two densities of states, one numbering the minima and another essentially giving the entropy of each minimum. Thus, each minimum is a cluster of states that may be reached from each other by not exciting all the way to the energy $E_{c}{ }^{118}$ Besides giving greater flexibility to the EME model, making it able to fit the frequency-dependent specificheat experiments, this approach also allows for the existence of $\beta$ relaxation as the process associated with intraminima transitions. ${ }^{118}$

\section{CONCLUSIONS}

A derivation of Bässler's random-walk model has been sketched, which emphasizes the potential importance of this model as a "canonical" or prototype phenomenologi- 
cal model for viscous liquids and the glass transition. The random-walk model views relaxation as a consequence of activated transport in a multidimensional rugged energy landscape. It is probably the simplest model of this type. The "derivation" of Sec. II traces the random-walk model back to Newton's equations for the molecules of one region. However, the derivation is in no way exact, which is clear just from the fact that the crystalline state of much lower energy than the supercooled liquid state is absent from the model.

It has been shown that the EME gives a good approximate description of the energy fluctuations of the random-walk model. The EME is solvable by a combination of analytical and numerical techniques (Appendix). This makes it possible to predict the behavior of the random-walk model for an arbitrary temperature time variation at very long times. Independently of its justification from the random-walk model, the EME may have a value of its own as a phenomenological model for viscous liquids and the glass transition. It incorporates true cooperativity and is consistent with statistical mechanics, while still being simple and solvable for realistic laboratory time scales. It is noteworthy that such a simple model leads to new predictions, like that there are two different types of glass transitions and that a wellannealed glass upon heating gives an anomalously large $\mathrm{x}$-ray scattering at intermediate times before equilibrium is reached.

\section{ACKNOWLEDGMENTS}

The author wishes to thank N. B. Olsen and T. Christensen for numerous illuminating discussions, and P. Borgstr $\varnothing \mathrm{m}$ and I. H. Pedersen for technical assistance. This work was supported by the Danish Natural Science Research Council.

\section{APPENDIX: SOLVING THE ENERGY MASTER EQUATION}

We first calculate how an initial nonequilibrium energy probability distribution at a fixed temperature converges to the canonical equilibrium distribution. ${ }^{43,79-82}$ To solve the EME numerically, it must be discretized. The energy axis is discretized into $N$ evenly spaced energies, $E_{1}<\cdots<E_{N}$. At low temperatures it is important to include large negative energies into the set of discrete energies, despite these lying far into the Gaussian tail. If one defines $\Gamma_{i} \equiv \Gamma\left(E_{i}\right), \quad P_{i} \equiv P\left(E_{i}, t\right) / C_{p}, \quad$ and $n_{i} \equiv n\left(E_{i}\right) / C_{n}$, where the normalization constants $C_{p}$ and $C_{n}$ are determined so that

$$
\sum_{j=1}^{N} P_{j}=1 ; \quad \sum_{j=1}^{N} n_{j}=1
$$

the EME Eq. (20) becomes upon discretization

$$
\frac{d P_{i}}{d t}=-\Gamma_{i} P_{i}+n_{i} \sum_{j=1}^{N} \Gamma_{j} P_{j} \quad(i=1, \ldots, N) .
$$

The normalization condition for the $n_{j}$ 's [Eq. (A1)] ensures probability conservation at all times.
At any temperature this equation may be solved by Laplace transformation. ${ }^{43,80-83}$ The Laplace transform of the function $P_{i}(t)$ in Eq. (A2), $\widetilde{P}_{i}(s)$, is as usual defined ${ }^{83}$ by

$$
\widetilde{P}_{i}(s)=\int_{0}^{\infty} P_{i}(t) e^{-s t} d t .
$$

Since the Laplace transform of the time derivative of a function $f(t)$ is $s \widetilde{f}(s)-f(0)$, Eq. (A2) becomes upon Laplace transforming

$$
s \widetilde{P}_{i}(s)-P_{i}(0)=-\Gamma_{i} \widetilde{P}_{i}(s)+n_{i} X(s),
$$

where $X(s)=\sum_{j=1}^{N} \Gamma_{j} \widetilde{P}_{j}(s)$. This equation determines $\widetilde{P}_{i}(s)$ from a knowledge of the initial probabilities $P_{i}(0)$. The probabilities $P_{i}(t)$ are calculated by the inverse Laplace transformation, ${ }^{83}$

$$
P_{i}(t)=\frac{1}{2 \pi i} \int_{-i \infty}^{+i \infty} \widetilde{P}_{i}(s) e^{s t} d s .
$$

Isolating $\widetilde{P}_{i}(s)$ from Eq. (A4) leads to

$$
\widetilde{P}_{i}(s)=\frac{P_{i}(0)}{s+\Gamma_{i}}+\frac{n_{i}}{s+\Gamma_{i}} X(s) .
$$

From this expression an equation for $X(s)$ is found by multiplying with $\Gamma_{i}$ on each side and summing:

$$
X(s)=\sum_{i=1}^{N} \frac{\Gamma_{i} P_{i}(0)}{s+\Gamma_{i}}+X(s) \sum_{i=1}^{N} \frac{n_{i} \Gamma_{i}}{s+\Gamma_{i}},
$$

or

$$
X(s)=\frac{\sum_{i=1}^{N}\left[\Gamma_{i} P_{i}(0) /\left(s+\Gamma_{i}\right)\right]}{1-\sum_{i=1}^{N}\left[n_{i} \Gamma_{i} /\left(s+\Gamma_{i}\right)\right]} .
$$

Using Eq. (A1) the denominator may be rewritten

$$
1-\sum_{i=1}^{N} \frac{n_{i}\left(\Gamma_{i}+s-s\right)}{s+\Gamma_{i}}=s \sum_{i=1}^{N} \frac{n_{i}}{s+\Gamma_{i}} .
$$

When this is substituted into Eq. (A8), Eq. (A6) becomes (changing the summation index from $i$ to $j$ )

$$
\widetilde{P}_{i}(s)=\frac{P_{i}(0)}{s+\Gamma_{i}}+\frac{n_{i}}{s\left(s+\Gamma_{i}\right)} \frac{\sum_{j=1}^{N}\left[\Gamma_{j} P_{j}(0) /\left(s+\Gamma_{j}\right)\right]}{\sum_{j=1}^{N}\left[n_{j} /\left(s+\Gamma_{j}\right)\right]} .
$$

From Eq. (A10) $P_{i}(t)$ may be calculated via Eq. (A5), where the integration contour in the complex plane lies to the right of all poles of $\widetilde{P}_{i}(s)$. The integral is evaluated by including an infinitely large semicircle surrounding the left half of the complex plane. This closes the integration contour and the residue theorem may be applied. For each $i$ there are $N$ poles which, due to the structure of the energy master equation, are the same for all $\widetilde{P}_{i}(s)$. There is one pole at $s=0$. The apparent singularities at $s=-\Gamma_{j}$, are all "removable", i.e., not real singularities. If $j=i$ this follows from the fact that. 


$$
\lim _{s \rightarrow-\Gamma_{i}}\left(s+\Gamma_{i}\right) \widetilde{P}_{i}(s)=P_{i}(0)+\frac{n_{i}}{\left(-\Gamma_{i}\right)} \frac{\Gamma_{i} P_{i}(0)}{n_{i}}=0,
$$

while for $j \neq i$ it follows from

$$
\lim _{s \rightarrow-\Gamma_{j}}\left(s+\Gamma_{j}\right) \widetilde{P}_{i}(s)=0+0 \frac{\Gamma_{j} P_{j}(0)}{n_{j}}=0 .
$$

Besides the $s=0$ pole there are poles whenever $s$ obeys

$$
\sum_{j=1}^{N} \frac{n_{j}}{s+\Gamma_{j}}=0
$$

This equation has $N-1$ solutions, each of which is a negative real number. The solutions are conveniently denoted by $s=-\omega_{k}$ and numbered such that

$$
\Gamma_{k}<\omega_{k}<\Gamma_{k+1} \quad(k=1, \ldots, N-1) .
$$

The $\omega$ 's are thus defined by

$$
\sum_{j=1}^{N} \frac{n_{j}}{\Gamma_{j}-\omega_{k}}=0 \quad(k=1, \ldots, N-1) .
$$

We next proceed to find the residues. At the pole $s=0$ the residue is given by

$$
\lim _{s \rightarrow 0} s \widetilde{P}_{i}(s)=0+\frac{n_{i}}{\Gamma_{i}} \frac{\sum_{j=1}^{N} P_{j}(0)}{\sum_{j=1}^{N}\left(n_{j} / \Gamma_{j}\right)}=\frac{n_{i} / \Gamma_{i}}{\sum_{j=1}^{N}\left(n_{j} / \Gamma_{j}\right)} .
$$

Since the quantity $n_{i} / \Gamma_{i} \propto n_{i} \exp \left(-\beta E_{i}\right)$ is proportional to the canonical equilibrium probability for the system having energy $E_{i}$, the residue at $s=0$ is simply the normalized equilibrium probability $P_{0, i}$ :

$$
\lim _{s \rightarrow 0} s \widetilde{P}_{i}(s)=P_{0, i} .
$$

Using the rule that the residue of a function of the form $f(z) / g(z)$ at a simple zero for $g(z)$ at $z=z_{0}$ is equal to $f\left(z_{0}\right) / g^{\prime}\left(z_{0}\right)$, one finds for the residues at $s=-\omega_{k}$

$$
\begin{aligned}
& \lim _{s \rightarrow-\omega_{k}}\left(s+\omega_{k}\right) \widetilde{P}_{i}(s) \\
&=0 \frac{P_{i}(0)}{\Gamma_{i}-\omega_{k}}+ \frac{n_{i}}{\left(-\omega_{k}\right)\left(\Gamma_{i}-\omega_{k}\right)} \\
& \times \frac{\sum_{j=1}^{N}\left[\Gamma_{j} P_{j}(0) /\left(\Gamma_{j}-\omega_{k}\right)\right]}{\sum_{j=1}^{N}\left[\left(-n_{j}\right) /\left(\Gamma_{j}-\omega_{k}\right)^{2}\right]} \\
&=\frac{n_{i}}{\omega_{k}\left(\Gamma_{i}-\omega_{k}\right)} A_{k},
\end{aligned}
$$

where

$$
A_{k}=\frac{\sum_{j=1}^{N}\left[\Gamma_{j} P_{j}(0) /\left(\Gamma_{j}-\omega_{k}\right)\right]}{\sum_{j=1}^{N}\left[n_{j} /\left(\Gamma_{j}-\omega_{k}\right)^{2}\right]}(k=1, \ldots, N-1)
$$

Having determined the residues, the integral Eq. (A5) is now easily calculated by the residue theorem:

$$
P_{i}(t)=P_{0, i}+\sum_{k=1}^{N-1} \frac{n_{i}}{\omega_{k}\left(\Gamma_{i}-\omega_{k}\right)} A_{k} e^{-\omega_{k} t} .
$$

The equations (A15), (A19), and (A20) give the solution of the energy master equation at a fixed temperature. As expected, the solution converges to the equilibrium solution as $t \rightarrow \infty$. The $\omega_{k}$ 's play the role of characteristic relaxation rates. Note that conservation of probability is ensured by virtue of Eq. (A15).

In the numerical implementation, the $\omega_{k}$ 's are determined from Eq. (A15) by the bisection method. Depending on the numerical precision large numerical errors may arise from the term $1 /\left(\Gamma_{k}-\omega_{k}\right)$ in Eqs. (A19) and (A20) at low energies where $\omega_{k}$ is extremely close to $\Gamma_{k}$; in this case one may use Eq. (A15) to approximate as follows:

$$
\frac{1}{\Gamma_{k}-\omega_{k}} \cong-\frac{1}{n_{k}} \sum_{j=1, j \neq k}^{N} \frac{n_{j}}{\Gamma_{j}-\omega_{k}} .
$$

Another problem that may arise is overflow. In the present work both these numerical problems were avoided by using the 80 bit floating point "extended" data type of Turbo Pascal. Alternatively, overflow problems may be avoided by the following procedure: The numbers $P_{j}, P_{j}(0), n_{j}, \Gamma_{k}, \omega_{k}$ are each represented by their logarithm. Each sum appearing in Eqs. (A15), (A19), and (A20) is evaluated by first identifying the leading term and then factorizing it. There remains a sum of terms less than one, each term being a product which is evaluated as, e.g., $a b=\exp [\ln (a)+\ln (b)]$.

By means of Eq. (A20) the master equation may be solved numerically at arbitrary long times at a fixed temperature with great accuracy. If the temperature changes in time, the above method is applied for time steps small enough that the temperature may be regarded as constant. In the solutions of the master equation plotted in Figs. 2-5 the energy axis was discretized into energies spaced $0.1 \sigma$ apart spanning an energy interval of $10 \sigma$, suitably placed on the energy axis depending on the problem. The solutions plotted in Fig. 2 were obtained from $2 \times 100$ time steps where the temperature is changed in each step. In two dimensions the percolation energy [Eq. (14)] is given by $E_{c}=0.235 \sigma$.
${ }^{1} \mathrm{G}$. Tammann, Der Glaszustand (Voss, Leipzig, 1933).

${ }^{2}$ W. Kauzmann, Chem. Rev. 43, 219 (1948).

${ }^{3}$ R. O. Davies and G. O. Jones, Adv. Phys. 2, 370 (1953).

${ }^{4} \mathrm{M}$. Goldstein, in Modern Aspects of the Vitreous State, edited by
J. D. Mackenzie (Butterworths Scientific, London, 1964), p. 90.

${ }^{5}$ C. A. Angell and W. Sichina, Ann. N.Y. Acad. Sci. 279, 53 (1976). 
${ }^{6}$ E. Donth, Glasübergang (Akademie-Verlag, Berlin, 1981).

${ }^{7}$ C. A. Angell, in Relaxations in Complex Systems, edited by K. L. Ngai and G. B. Wright (Government Printing Office, Washington, DC, 1985), p. 3.

${ }^{8} \mathrm{~S}$. Brawer, Relaxation in Viscous Liquids and Glasses (American Ceramic Society, Columbus, OH, 1985).

${ }^{9} \mathrm{G}$. W. Scherer, Relaxation in Glass and Composites (Wiley, New York, 1986).

${ }^{10}$ J. Jäckle, Rep. Prog. Phys. 49, 171 (1986).

${ }^{11}$ C. A. Angell, J. Non-Cryst. Solids 131-133, 13 (1991).

${ }^{12}$ F. Yonezawa, in Solid State Physics: Advances in Research and Applications, edited by $\mathrm{H}$. Ehrenreich and D. Turnbull (Academic, New York, 1991), Vol. 45, p. 179.

${ }^{13}$ A. Hunt, J. Non-Cryst. Solids 160, 183 (1993).

${ }^{14}$ I. M. Hodge, J. Non-Cryst. Solids 169, 211 (1994).

${ }^{15} \mathrm{H}$. Rawson, Properties and Applications of Glass (Elsevier, Amsterdam, 1980).

${ }^{16}$ C. A. Angell, J. Phys. Chem. 70, 2793 (1966).

${ }^{17}$ A. J. Kovacs, Fortschr. Hochpoly.-Forsch. 3, 394 (1963).

${ }^{18}$ J. D. Ferry, Viscoelastic Properties of Polymers, 3rd ed. (Wiley, New York, 1980).

${ }^{19}$ H. S. Chen, Rep. Prog. Phys. 43, 353 (1980).

${ }^{20}$ A. J. Barlow, J. Lamb, and A. J. Matheson, Proc. R. Soc. London, Ser. A 292, 322 (1966).

${ }^{21}$ G. Harrison, The Dynamic Properties of Supercooled Liquids (Academic, New York, 1976).

${ }^{22}$ C. A. Angell, J. H. R. Clarke, and L. V. Woodcock, Adv. Chem. Phys. 48, 397 (1981).

${ }^{23}$ J.-P. Hansen, Physica A 201, 138 (1993).

${ }^{24}$ K. Binder and A. P. Young, Rev. Mod. Phys. 58, 801 (1986).

${ }^{25}$ V. S. Dotsenko, Usp. Fiz. Nauk 163, 1 (1993) [Phys. Usp. 36, 455 (1993)].

${ }^{26}$ W. Götze and L. Sjögren, Rep. Prog. Phys. 55, 241 (1992).

${ }^{27}$ S. P. Das and G. F. Mazenko, Phys. Rev. A 34, 2265 (1986).

${ }^{28}$ Second International Discussion Meeting on Relaxations in Complex Systems, Alicante, Spain, 1993, edited by K. L. Ngai, E. Riande, and G. B. Wright [J. Non-Cryst. Solids 172174, 1-1457 (1994).

${ }^{29}$ G. W. Scherer, J. Non-Cryst. Solids 123, 75 (1990).

${ }^{30}$ C. A. Angell, J. Phys. Chem. Solids 49, 863 (1988).

${ }^{31}$ J. Jäckle, in Structure, Relaxation, and Physical Aging of Glassy Polymers, edited by R. J. Roe and J. M. O'Reilly, MRS Symposia Proceedings No. 215 (Materials Research Society, Pittsburgh, 1991), p. 151.

${ }^{32}$ B. Derrida, Phys. Rev. Lett. 45, 79 (1980).

${ }^{33}$ G. H. Fredrickson, Ann. Rev. Phys. Chem. 39, 149 (1988).

${ }^{34}$ T. A. Weber, G. H. Fredrickson, and F. H. Stillinger, Phys. Rev. B 34, 7641 (1986).

${ }^{35}$ J. H. Gibbs and E. A. DiMarzio, J. Chem. Phys. 28, 373 (1958).

${ }^{36}$ G. Adam and J. H. Gibbs, J. Chem. Phys. 43, 139 (1965).

${ }^{37}$ P. D. Gujrati and M. Goldstein, J. Chem. Phys. 74, 2596 (1981).

${ }^{38}$ C. A. Angell and K. J. Rao, J. Chem. Phys. 57, 470 (1972).

${ }^{39}$ M. Goldstein, Faraday Symp. Chem. Soc. 6, 7 (1972).

${ }^{40}$ G. S. Grest and M. H. Cohen, Adv. Chem. Phys. 48, 455 (1981).

${ }^{41}$ N. G. van Kampen, Stochastic Processes in Physics and Chemistry (North-Holland, Amsterdam, 1981).

${ }^{42}$ M. Goldstein, J. Chem. Phys. 51, 3728 (1969).

${ }^{43}$ S. A. Brawer, J. Chem. Phys. 81, 954 (1984).

${ }^{44}$ J. C. Dyre, Phys. Rev. Lett. 58, 792 (1987).

${ }^{45}$ H. Bässler, Phys. Rev. Lett. 58, 767 (1987).

${ }^{46}$ R. Richert and H. Bässler, J. Phys.: Condens. Matter 2, 2273
(1990).

${ }^{47}$ J. W. Haus and K. W. Kehr, Phys. Rep. 150, 263 (1987).

48 J. C. Dyre, Phys. Rev. B 49, 11709 (1994).

${ }^{49}$ H. Bässler, in Advances in Disordered Semiconductors, edited by H. Fritzsche and M. Pollak (World Scientific, Singapore, 1990), p. 491.

${ }^{50}$ J. H. Simmons and P. B. Macedo, J. Res. Nat. Bureau Stand. 75A, 175 (1971).

${ }^{51}$ H. Tweer, J. H. Simmons, and P. B. Macedo, J. Chem. Phys. 54, 1952 (1971).

${ }^{52}$ T. A. Litovitz, J. Chem. Phys. 20, 1088 (1952).

${ }^{53}$ V. I. Arkhipov and H. Bässler, J. Phys. Chem. 98, 662 (1994).

${ }^{54}$ A. Hunt, Int. J. Theor. Phys. B 8, 855 (1994).

${ }^{55}$ W. Kauzmann, Rev. Mod. Phys. 14, 12 (1942).

${ }^{56}$ T. Alfrey, Mechanical Properties of High Polymers (Interscience, New York, 1948).

${ }^{57} \mathrm{G}$. Williams, in Dielectric and Related Molecular Processes, edited by M. Davies (Chemical Society, London, 1975), p. 151.

${ }^{58}$ E. Donth, J. Non-Cryst. Solids 53, 325 (1982).

${ }^{59}$ F. H. Stillinger, J. Chem. Phys. 89, 6461 (1988).

${ }^{60}$ R. V. Chamberlin, Phys. Rev. B 48, 15638 (1993).

${ }^{61}$ G. Wahnström, Phys. Rev. A 44, 3752 (1991).

${ }^{62}$ R. D. Mountain and D. Thirumalai, Phys. Rev. A 45, R3380 (1992).

${ }^{63}$ F. H. Stillinger and T. A. Weber, Science 225, 983 (1984).

${ }^{64}$ F. H. Stillinger, Phys. Rev. B 41, 2409 (1990).

${ }^{65} \mathrm{M}$. Doi and S. F. Edwards, The Theory of Polymer Dynamics (Clarendon, Oxford, 1986).

${ }^{66}$ W. Hess and R. Klein, Adv. Phys. 32, 173 (1983).

${ }^{67}$ H. Löwen, J.-P. Hansen, and J.-N. Roux, Phys. Rev. A 44, 1169 (1991).

${ }^{68}$ H. Eyring, J. Chem. Phys. 4, 283 (1936).

${ }^{69}$ P. G. Wolynes, Acc. Chem. Res. 25, 513 (1992).

${ }^{70}$ M. B. Isichenko, Rev. Mod. Phys. 64, 961 (1992).

${ }^{71}$ S. Kirkpatrick, Rev. Mod. Phys. 45, 574 (1973).

${ }^{72}$ K. Huang, Statistical Mechanics (Wiley, New York, 1963).

${ }^{73}$ A. Miller and E. Abrahams, Phys. Rev. 120, 745 (1960).

${ }^{74}$ V. Ambegaokar, B. I. Halperin, and J. S. Langer, Phys. Rev. B 4, 2612 (1971).

${ }^{75}$ B. I. Shklovskii and A. L. Efros, Zh. Eksp. Teor. Fiz. 60, 867 (1971) [Sov. Phys. JETP 33, 468 (1971)].

${ }^{76} \mathrm{D}$. Stauffer and A. Aharony, Introduction to Percolation Theory, 2nd ed. (Taylor and Francis, London, 1992).

${ }^{77}$ V. I. Arkhipov, M. S. Iovu, A. I. Rudenko, and S. D. Shutov, Phys. Status Solidi A 54, 67 (1979).

${ }^{78}$ J. Orenstein, M. A. Kastner, and V. Vaninov, Philos. Mag. B 46, 23 (1982).

${ }^{79}$ R. E. Robertson, R. Simha, and J. G. Curro, Macromolecules 17, 911 (1984).

${ }^{80}$ G. J. M. Koper and H. J. Hilhorst, Europhys. Lett. 3, 1213 (1987).

${ }^{81}$ E. I. Shakhnovich and A. M. Gutin, Europhys. Lett. 9, 569 (1989).

82J. C. Dyre (unpublished).

${ }^{83}$ G. Arfken, Mathematical Methods for Physicists (Academic, New York, 1985).

${ }^{84}$ C. T. Moynihan, A. J. Easteal, M. A. DeBolt, and J. Tucker, J. Am. Ceram. Soc. 59, 12 (1976).

${ }^{85}$ J. C. Dyre, Key Eng. Mater. 13-15, 501 (1987).

${ }^{86}$ W. Tschöp and R. Schilling, Phys. Rev. E 48, 4221 (1993).

${ }^{87}$ J. C. Dyre, J. Appl. Phys. 64, 2456 (1988).

${ }^{88}$ H. Frauenfelder, S. G. Sligar, and P. G. Wolynes, Science 254, 1598 (1991). 
${ }^{89}$ C. Aslangul, N. Pottier, and D. Saint-James, Physica A 164, 52 (1990).

${ }^{90}$ R. Tao, Phys. Rev. A 43, 5284 (1991).

${ }^{91}$ A. B. Kolomeisky and E. B. Kolomeisky, Phys. Rev. A 45, R5327 (1992).

${ }^{92}$ M. D. Bal'makov, Fiz. Khim. Stekla 12, 527 (1986) [Sov. J. Glass Phys. Chem. 12, 279 (1986)].

${ }^{93}$ R. D. Young, J. Chem. Phys. 98, 2488 (1993).

${ }^{94}$ Y. Hiwatari, J. Chem. Phys. 76, 5502 (1982).

${ }^{95}$ A. Hunt, Solid State Commun. 84, 701 (1992).

${ }^{96}$ M. F. Thorpe, J. Non-Cryst. Solids 57, 355 (1983).

${ }^{97}$ M. Silver, G. Schönherr, and H. Bässler, Phys. Rev. Lett. 48 , 352 (1982).

${ }^{98}$ H. Bässler, Prog. Colloid Polym. Sci. 80, 35 (1989).

${ }^{99}$ M. Goldstein, J. Chem. Phys. 64, 4767 (1976).

${ }^{100}$ B. Ries, H. Bässler, M. Grünewald, and B. Movaghar, Phys. Rev. B 37, 5508 (1988).

${ }^{101}$ A. R. Dexter and A. J. Matheson, Adv. Mol. Relaxation Processes 2, 251 (1972).

${ }^{102}$ S. V. Nemilov, Fiz. Khim. Stekla 2, 193 (1976) [Sov. J. Glass Phys. Chem. 2, 187 (1976)].

${ }^{103}$ S. V. Nemilov, Fiz. Khim. Stekla 4, 129 (1978) [Sov. J. Glass
Phys. Chem. 4, 113 (1978)].

${ }^{104}$ T. A. Vilgis, Phys. Rev. B 47, 2882 (1993).

${ }^{105}$ R. Böhmer, J. Non-Cryst. Solids 172-174, 628 (1994).

${ }^{106} \mathrm{G}$. M. Bartenev, The Structure and Mechanical Properties of Inorganic Glasses (Wolters-Noordhoff, Groningen, 1970).

${ }^{107}$ M. R. J. Gibbs, J. E. Evetts, and J. A. Leake, J. Mater. Sci. 18, 278 (1983).

${ }^{108}$ A. van den Beukel, S. van der Zwaag, and A. L. Mulder, Acta Metall. 32, 1895 (1984).

${ }^{109}$ O. V. Mazurin, J. Non-Cryst. Solids 25, 130 (1977).

${ }^{110}$ G. P. Johari and E. Whalley, Faraday Symp. Chem. Soc. 6, 23 (1972).

${ }^{111}$ R. Zwanzig, J. Chem. Phys. 88, 5831 (1988).

${ }^{112}$ T. Christensen and N. B. Olsen, Phys. Rev. B 49, 15396 (1994); (unpublished).

${ }^{113}$ S. V. Nemilov, Fiz. Khim. Stekla 11, 146 (1985) [Sov. J. Glass Phys. Chem 11, 97 (1985)].

${ }^{114}$ K. D. Jensen and J. Nielsen (unpublished).

${ }^{115}$ T. Christensen, J. Phys. (Paris) Colloq. 46, C8-635 (1985).

${ }^{116}$ N. O. Birge and S. R. Nagel, Phys. Rev. Lett. 54, 2674 (1985).

${ }^{117}$ J. C. Dyre (unpublished).

${ }^{118}$ V. Rosato and G. Williams, Adv. Mol. Relaxation Processes 20, 233 (1981). 